\title{
On approximate solutions to the Neumann elliptic boundary value problem with non-linearity of exponential type
}

\author{
Peter I Kogut ${ }^{1 *}$, Rosanna Manzo ${ }^{2}$ (i) and Anna O Putchenko'
}

Correspondence: p.kogut@i.ua
${ }^{1}$ Department of Differential
Equations, Dnipropetrovsk National
University, Gagarin av., 72,49010
Dnipro, Ukraine
Full list of author information is
available at the end of the article

available at the end of the article

\begin{abstract}
We discuss the existence of weak solutions to one class of Neumann boundary value problems (BVP) for non-linear elliptic equations. We introduce a special family of perturbed optimal control problems (OCPs) where the class of fictitious controls is closely related with the properties of the distribution in the right-hand side of the elliptic equation, and we show that optimal solutions of such problems allow one to attain (in the limit) some approximate solutions as the parameter of perturbation $\varepsilon>0$ tends to zero. The main questions we discuss in this paper touch on the solvability of perturbed OCPs, the uniqueness of their solutions, the asymptotic properties of optimal pairs as the perturbation parameter $\varepsilon>0$ tends to zero, and deriving of optimality conditions for the perturbed OCPs. As a consequence, we obtain the sufficient conditions of the existence of weak solutions to the given class of non-linear Neumann BVPs and propose a way for their approximation.
\end{abstract}

MSC: Primary 5J25; 35J60; secondary 49J20; 49K20; 58J37

Keywords: existence result; semilinear elliptic equation; fictitious control; perturbation approach

\section{Introduction}

In this paper we are concerned with the following Neumann boundary value problem (BVP):

$$
\begin{aligned}
& -\Delta y+y=f(y)+g \text { in } \Omega, \\
& \frac{\partial y}{\partial v}=0 \quad \text { on } \partial \Omega,
\end{aligned}
$$

where $f(y)=F^{\prime}(y), F \in C^{1}(K)$ for any compact set $K \subset \mathbb{R}, F(z) \geq F(0) \exp \left(C_{F}^{-1} z\right)$ for all $z \in \mathbb{R}, \frac{\partial}{\partial v}$ is the outward normal derivative, and $g \in L^{p}(\Omega)$ for some $2<p<\infty$ is a given distribution.

It is well known that the indicated BVP is ill-posed in general. It means that there is no reason to assert the existence of weak solutions to (1.1)-(1.2) for a given $g \in L^{p}(\Omega)$, or to suppose that such solution, even if it exists, is unique (see, for instance, Gelfand [1], Crandall and Rabinowitz [2], Mignot and Puel [3], Gallouët, Mignot and Puel [4], Fujita

(c) The Author(s) 2016. This article is distributed under the terms of the Creative Commons Attribution 4.0 International License (http://creativecommons.org/licenses/by/4.0/), which permits unrestricted use, distribution, and reproduction in any medium, provided you give appropriate credit to the original author(s) and the source, provide a link to the Creative Commons license, and indicate if changes were made. 
[5], Pinsky [6], Ferreira, De Pablo, Vazquez [7], Dolbeault and Stańczy [8]). At the same time, the BVPs like (1.1)-(1.2) appear in many contexts: in the study of stellar structures [9], in combustion theory for the chemical reactors [10], etc.

The aim of this article is to discuss the existence of weak solutions to the Neumann boundary value problem (1.1)-(1.2) and propose a way for their approximation. The characteristic feature of the indicated BVP (1.1)-(1.2) is the fact that because of the property of non-linearity of $F(y)$, we have no prior estimate for the weak solutions in the standard functional space. Moreover, since we cannot assert that the BVP (1.1)-(1.2) admits at least one solution for a given $g \in L^{p}(\Omega)$, our main intention is to show that the original BVP possesses the so-called approximate weak solutions. To do so, we define the approximate solutions as the weak solutions to the problem (1.1)-(1.2) with special choice of the distribution $g^{*} \in \bar{\Lambda}$ which must be close (in some sense) to the original one $g$. The key point in this approach is the construction of the set of feasible distributions $\bar{\Lambda}$. As we will show later on, this set has a rather complicate structure. So, it is not an easy matter to touch on the choice of $g^{*} \in \bar{\Lambda}$ directly. In view of this, we introduce a special family of perturbed optimal control problems (OCPs) $\left\langle\inf _{(u, y) \in \Xi_{\Delta}} J_{\varepsilon}(u, y)\right\rangle$, where

$$
J_{\varepsilon}(u, y)=\frac{\varepsilon}{2}\|\Delta y\|_{L^{2}(\Omega)}^{2}+\frac{1}{p}\|g-u\|_{L^{p}(\Omega)}^{p}
$$

$u \in L^{p}(\Omega)$ is the fictitious control, and $\Xi_{\Delta}$ is the set of feasible pairs $(u, y)$ (admissible control and the 'corresponding' weak solution) restricted by the constraints $J_{\varepsilon}(u, y)<+\infty$, $f(y) \in L^{1}(\Omega)$, and related by the integral identity

$$
\int_{\Omega}[(\nabla y, \nabla \varphi)+y \varphi] d x=\int_{\Omega} f(y) \varphi d x+\int_{\Omega} u \varphi d x, \quad \forall \varphi \in C_{0}^{\infty}\left(\mathbb{R}^{N}\right) .
$$

The crucial questions we discuss in this paper are about solvability of perturbed OCPs, uniqueness of their solutions, and asymptotic properties of optimal pairs as the perturbation parameter $\varepsilon>0$ tends to zero. The plan of the paper is as follows. In Section 2 we give the formal statement of the boundary value problem and establish the necessary background to its study. Following the ideas of Casas, Kavian, and Puel in [11], we study in Section 3 some auxiliary properties of the weak solutions to the Neumann problem (1.1)-(1.2). In particular, we show that a prior estimate for the weak solutions in $H^{1}(\Omega)$ can be derived if only such solutions have $L^{2}(\Omega)$-bounded Laplacian. The key result of this section is Proposition 3.5, which gives reasons to suppose that the set of weak solutions with $L^{2}(\Omega)$-bounded Laplacian is not weakly closed in $H^{1}(\Omega)$ in general. In Section 4, we consider the sequence of parametrized OCPs associated with the original BVP (1.1)-(1.2) and show that each of this problem has a unique optimal control in spite of the fact that the corresponding sets of fictitious controls are not necessary closed in the $L^{p}$-topology. The variational properties of optimal pairs to the parametrized OCPs and their asymptotic behavior are studied in Section 5 . It has been shown that such sequences are sequentially compact in the weak topology of $L^{p}(\Omega) \times H^{1}(\Omega)$ and each of their cluster points can be interpreted as an approximation solution to the original BVP. In the last section we investigate the optimality conditions to the fictitious OCPs.

Thus, we derive the sufficient conditions of the existence of weak solutions to the class of non-linear Neumann BVP (1.1)-(1.2) with $g \in L^{p}(\Omega)$ (where $p>2$ ) and give a practical approach to the approximation of such solutions (for the details we refer to Theorem 7.2). It 
seems to us, following the same way the existence can be studied of approximate solutions to BVP (1.1)-(1.2) provided the distribution $g$ is in $\mathrm{BV}(\Omega) \cap L^{2}(\Omega)$. However, as to the case when $g \in L^{2}(\Omega)$ and $g \notin L^{2+\delta}(\Omega)$ for any $\delta>0$, it apparently remains an open question now.

\section{Statement of the problem and some preliminaries}

Let $\Omega$ be a bounded open connected subset of $\mathbb{R}^{N}(N>2)$. We assume that the boundary $\partial \Omega$ is of the class $C^{1,1}$ or $\Omega$ is a convex domain with Lipschitzian boundary. So, the unit outward normal $v=v(x)$ is well defined for $\mathcal{H}^{N-1}$-a.a. $x \in \partial \Omega$, where a.a. means here with respect to the $(N-1)$-dimensional Hausdorff measure $\mathcal{H}^{N-1}$. Throughout the paper we assume that $\Omega$ is star-shaped with respect to some interior point $x_{0}$, i.e. $\left(\sigma-x_{0}, v(\sigma)\right) \geq 0$ for $\mathcal{H}^{N-1}$-a.a. $\sigma \in \partial \Omega$.

Let $F: \mathbb{R} \rightarrow(0,+\infty)$ be a mapping such that $F \in C_{\text {loc }}^{1}(\mathbb{R})$ and there exists a constant $C_{F}>0$ satisfying

$$
F(z) \leq C_{F} F^{\prime}(z), \quad \forall z \in \mathbb{R}
$$

that is, in the following we may suppose that $F(z) \geq F(0) \exp \left(C_{F}^{-1} z\right)$ over $\mathbb{R}$. Hereinafter the condition $\varphi \in C_{\text {loc }}^{k}(\mathbb{R})$ means that we deal with a function $\varphi: \mathbb{R} \rightarrow \mathbb{R}$ satisfying $\varphi \in C^{k}(K)$ for any compact set $K \subset \mathbb{R}$.

Let $p(2<p<\infty)$ be a given real number and let $g \in L^{p}(\Omega)$ be a given distribution. By $H^{1}(\Omega)$ we denote the Sobolev space as the closure of $C_{0}^{\infty}\left(\mathbb{R}^{N}\right)$ with respect to the norm $\|y\|_{H^{1}(\Omega)}=\left(\int_{\Omega}\left(y^{2}+|\nabla y|^{2}\right) d x\right)^{1 / 2}$. Let $\left(H^{1}(\Omega)\right)^{*}$ be the dual space to $H^{1}(\Omega)$.

In order to give a precise meaning to the solution to BVP (1.1)-(1.2) and indicate its characteristic properties, we begin with the following concept.

Definition 2.1 We say that a function $y=y(g)$ is a weak solution to the boundary value problem (1.1)-(1.2) for a given distribution $g \in L^{p}(\Omega)$ with $p>2$ if $y \in H^{1}(\Omega), y$ belongs to the class of functions

$$
Y=\left\{y \in H^{1}(\Omega) \mid f(y) \in L^{1}(\Omega)\right\}
$$

and the integral identity

$$
\int_{\Omega}[(\nabla y, \nabla \varphi)+y \varphi] d x=\int_{\Omega} f(y) \varphi d x+\int_{\Omega} g \varphi d x
$$

holds for every test function $\varphi \in C_{0}^{\infty}\left(\mathbb{R}^{N}\right)$.

As was indicated before, it is unknown whether the original BVP admits at least one weak solution in the sense of Definition 2.1 for a given distribution $g \in L^{p}(\Omega)$. Moreover, as follows from (2.2), the continuity of the form $[y, \varphi]_{f}:=\int_{\Omega} f(y) \varphi d x$ on the set $Y \subset H^{1}(\Omega)$ is not evident. This motivates us to introduce the following set.

Definition 2.2 We say that an element $y \in H^{1}(\Omega)$ belongs to the set $H_{f}$ if

$$
\left|\int_{\Omega} f(y) \varphi d x\right| \leq c(y)\left(\int_{\Omega} \varphi^{2} d x+\int_{\Omega}|\nabla \varphi|^{2} d x\right)^{1 / 2}, \quad \forall \varphi \in C_{0}^{\infty}\left(\mathbb{R}^{N}\right)
$$

with some constant depending on $y$. 
As a result, we have: if $y \in H_{f}$ then $y \in Y$. Moreover, in this case the mapping $\varphi \mapsto[y, \varphi]_{f}$ can be defined for all $\varphi \in H^{1}(\Omega)$ using (2.4) and the standard rule

$$
[y, \varphi]_{f}=\lim _{\varepsilon \rightarrow 0}\left[y, \varphi_{\varepsilon}\right]_{f}
$$

where $\left\{\varphi_{\varepsilon}\right\}_{\varepsilon>0} \subset C_{0}^{\infty}\left(\mathbb{R}^{N}\right)$ and $\varphi_{\varepsilon} \rightarrow \varphi$ strongly in $H^{1}(\Omega)$. In particular, if $y \in H_{f}$, then we can define the value $[y, y]_{f}$ and this one is finite for every $y \in H_{f}$, although the 'integrand' $y f(y)$ does not need to be integrable on $\Omega$, in general. Taking this fact into account, we immediately arrive at the following conclusion.

Proposition 2.3 If $g \in L^{p}(\Omega)$ is a given distribution and $y \in H_{f}$ is a weak solution to BVP (1.1)-(1.2) in the sense of Definition 2.1, then $y$ satisfies the energy equality

$$
\int_{\Omega}|\nabla y|^{2} d x+\int_{\Omega} y^{2} d x=[y, y]_{f}+\int_{\Omega} g y d x
$$

We note that by the initial assumptions and Hölder's inequality, this relation makes sense because

$$
\left|\int_{\Omega} g y d x\right| \leq\|g\|_{L^{2}(\Omega)}\|y\|_{L^{2}(\Omega)} \leq|\Omega|^{\frac{p-2}{2 p}}\|g\|_{L^{p}(\Omega)}\|y\|_{H^{1}(\Omega)}<+\infty
$$

However, since it is unknown whether the value $[y, y]_{f}$ preserves a constant sign for all $y \in H_{f}$, it follows that we cannot make use of the energy equality (2.6) in order to derive a prior estimate in $\|\cdot\|_{H^{1}(\Omega)}$-norm for the weak solutions. In particular, to specify the term $[y, y]_{f}$ we can use the following result.

Lemma 2.4 Let $y \in Y$ be a weak solution to BVP (1.1)-(1.2). Then $y \in H_{f}, f(y) \in\left(H^{1}(\Omega)\right)^{*}$, and

$$
[y, z]_{f}=\langle f(y), z\rangle_{\left(H^{1}(\Omega)\right)^{*} ; H^{1}(\Omega)}=\int_{\Omega} z f(y) d x, \quad \forall z \in H^{1}(\Omega),
$$

i.e. $z f(y) \in L^{1}(\Omega)$ for every $z \in H^{1}(\Omega)$.

Remark 2.5 Here, $\left(H^{1}(\Omega)\right)^{*}$ stands for the dual space of $H^{1}(\Omega)$ and, hence, $\left(H^{1}(\Omega)\right)^{*}$ can be identified with the direct sum $H^{-1}(\Omega) \oplus H^{-\frac{1}{2}}(\partial \Omega)$.

Proof Following the definition of the weak solution, we have (see (2.3))

$$
\begin{aligned}
\int_{\Omega} f(y) \varphi d x & \leq\left|\int_{\Omega}(\nabla y, \nabla \varphi) d x\right|+\left|\int_{\Omega} y \varphi d x\right|+\left|\int_{\Omega} g \varphi d x\right| \\
& \leq 2\|y\|_{H^{1}(\Omega)}\|\varphi\|_{H^{1}(\Omega)}+\|g\|_{L^{2}(\Omega)}\|\varphi\|_{L^{2}(\Omega)} \\
& \stackrel{\text { by (2.7) }}{\leq}\left(2\|y\|_{H^{1}(\Omega)}+|\Omega|^{\frac{p-2}{2 p}}\|g\|_{L^{p}(\Omega)}\right)\|\varphi\|_{H^{1}(\Omega)}, \quad \forall \varphi \in C_{0}^{\infty}\left(\mathbb{R}^{N}\right) .
\end{aligned}
$$

Hence, $y \in H_{f}$ by Definition 2.2. 
Let $z \in H^{1}(\Omega) \cap L^{\infty}(\Omega)$ be an arbitrary element. Since $f(y) \in L^{1}(\Omega)$, it follows that the term $\int_{\Omega} z f(y) d x$ is well defined. Let $\left\{\varphi_{\varepsilon}\right\}_{\varepsilon>0} \subset C^{\infty}(\bar{\Omega})$ be a sequence such that $\varphi_{\varepsilon} \rightarrow z$ in $H^{1}(\Omega)$. In view of our assumptions, it is plausible to suppose that

$$
\sup _{\varepsilon>0}\left\|\varphi_{\varepsilon}\right\|_{L^{\infty}(\Omega)}<+\infty \quad \text { and } \quad \varphi_{\varepsilon} \stackrel{*}{\rightarrow} z \quad \text { in } L^{\infty}(\Omega) .
$$

Hence, using the fact that $y \in H_{f}$, we get

$$
\int_{\Omega} z f(y) d x=\lim _{\varepsilon \rightarrow 0} \int_{\Omega} \varphi_{\varepsilon} f(y) d x=\lim _{\varepsilon \rightarrow 0}\left[y, \varphi_{\varepsilon}\right]_{f} \stackrel{\text { by }}{=}[2.5)[y, z]_{f}
$$

Thus, we arrive at equation (2.8) for each $z \in H^{1}(\Omega) \cap L^{\infty}(\Omega)$.

Let us take now $z \in H^{1}(\Omega)$ such that $z \geq 0$ almost everywhere in $\Omega$. For every $\varepsilon>0$, let $T_{\varepsilon}: \mathbb{R} \rightarrow \mathbb{R}$ be the truncation operator defined by

$$
T_{\varepsilon}(s)=\max \left\{\min \left\{s, \varepsilon^{-1}\right\},-\varepsilon^{-1}\right\} .
$$

The following property of $T_{\varepsilon}$ is well known (see [12]): If $z \in H^{1}(\Omega)$ then

$$
T_{\varepsilon}(z) \in L^{\infty}(\Omega) \cap H^{1}(\Omega) \quad \forall \varepsilon>0 \quad \text { and } \quad T_{\varepsilon}(z) \rightarrow z \quad \text { in } H^{1}(\Omega) \text { as } \varepsilon \rightarrow 0 .
$$

Hence, $T_{\varepsilon}(z) \rightarrow z$ almost everywhere in $\Omega$. Since

$$
T_{\varepsilon}(z) f(y) \stackrel{\text { by }(2.1)}{\geq} \frac{1}{C_{F}} T_{\varepsilon}(z) F(y)>0 \quad \text { in } \Omega,
$$

it follows that $\left\{T_{\varepsilon}(z) f(y)\right\}_{\varepsilon>0}$ is a pointwise non-decreasing sequence, and $T_{\varepsilon}(z) f(y) \rightarrow z f(y)$ for almost all $x \in \Omega$. Therefore, by the monotone convergence theorem, $z f(y)$ is a measurable function on $\Omega$, and

$$
\lim _{\varepsilon \rightarrow 0} \int_{\Omega} T_{\varepsilon}(z) f(y) d x=\int_{\Omega} z f(y) d x .
$$

Thus, (2.8) holds true for each $z \in H^{1}(\Omega)$ such that $z \geq 0$.

As for a general case, i.e. $z \in H^{1}(\Omega)$, it is enough to note that $z=z^{+}-z^{-}$with $z^{+}, z^{-} \in$ $H^{1}(\Omega)$ and $z^{+}, z^{-} \geq 0$ in $\Omega$, where

$$
z^{+}:=\max \{z, 0\}, \quad z^{-}:=\max \{-z, 0\} .
$$

To complete the proof, it remains to observe that

$$
\begin{aligned}
& \int_{\Omega} z f(y) d x \stackrel{\text { by }}{\stackrel{(2.12)}{=}} \lim _{\varepsilon \rightarrow 0} \int_{\Omega} \varphi_{\varepsilon} f(y) d x \\
& \stackrel{\text { by (2.9) }}{\leq} \lim _{\varepsilon \rightarrow 0}\left(2\|y\|_{H^{1}(\Omega)}+|\Omega|^{\frac{p-2}{2 p}}\|g\|_{L^{p}(\Omega)}\right)\left\|\varphi_{\varepsilon}\right\|_{H^{1}(\Omega)} \\
& \quad\left(\text { by the strong convergence of } \varphi_{\varepsilon} \rightarrow z \text { in } H^{1}(\Omega)\right) \\
&=\left(2\|y\|_{H^{1}(\Omega)}+|\Omega|^{\frac{p-2}{2 p}}\|g\|_{L^{p}(\Omega)}\right)\|z\|_{H^{1}(\Omega)}
\end{aligned}
$$


holds true for an arbitrary element $z \in H^{1}(\Omega)$. As a result, we have

$$
\begin{aligned}
& f(y) \in\left(H^{1}(\Omega)\right)^{*}, \quad\langle f(y), z\rangle_{\left(H^{1}(\Omega)\right)^{*} ; H^{1}(\Omega)}=\int_{\Omega} z f(y) d x, \quad \forall z \in H^{1}(\Omega), \\
& \text { and }\|f(y)\|_{\left(H^{1}(\Omega)\right)^{*}} \leq\left(2\|y\|_{H^{1}(\Omega)}+|\Omega|^{\frac{p-2}{2 p}}\|g\|_{L^{p}(\Omega)}\right) .
\end{aligned}
$$

As a direct consequence of this lemma and Proposition 2.3, we have the following result.

Corollary 2.6 Let $g \in L^{p}(\Omega)(p>2)$ be a given distribution and let $y \in H^{1}(\Omega)$ be a weak solution to BVP (1.1)-(1.2) in the sense of Definition 2.1. Then the energy equality for $y$ takes the form

$$
\int_{\Omega}|\nabla y|^{2} d x+\int_{\Omega}|y|^{2} d x=\int_{\Omega} y f(y) d x+\int_{\Omega} g y d x
$$

\section{Some auxiliary results and prior estimates}

In this section we deal with some extra properties of the weak solutions to the boundary value problem (1.1)-(1.2). In some aspects we follow the ideas of Casas, Kavian, and Puel [11] where the Dirichlet boundary value problem with exponential non-linearity has been considered.

Proposition 3.1 Let $y \in H^{1}(\Omega)$ be a weak solution to BVP (1.1)-(1.2) such that $\Delta y \in L^{2}(\Omega)$. Then

$$
\left(\frac{N}{2}-1\right) \int_{\Omega}|\nabla y|^{2} d x+\frac{N}{2} \int_{\Omega}|y|^{2} d x \leq N \int_{\Omega} F(y) d x-\int_{\Omega} g\left(x-x_{0}, \nabla y\right) d x,
$$

where $x_{0} \in$ int $\Omega$ is a point such that $\left(\sigma-x_{0}, v(\sigma)\right) \geq 0$ for almost all $\sigma \in \partial \Omega$, and $\nu(\sigma)$ denotes the outward unit normal vector to $\partial \Omega$ at the point $\sigma$.

Proof In view of the initial assumptions, we have $-\Delta y+y-g \in L^{2}(\Omega)$. Hence, $f(y) \in L^{2}(\Omega)$ and, therefore, the integral identity (2.3) makes sense for any test function $\varphi \in L^{2}(\Omega)$. Let us consider $\varphi:=\left(x-x_{0}, \nabla y\right) \in L^{2}(\Omega)$ as this function. Then (2.3) implies the relation

$$
\begin{gathered}
\int_{\Omega}\left(\nabla y, \nabla\left(x-x_{0}, \nabla y\right)\right) d x+\int_{\Omega} y\left(x-x_{0}, \nabla y\right) d x \\
=\int_{\Omega} f(y)\left(x-x_{0}, \nabla y\right) d x+\int_{\Omega} g\left(x-x_{0}, \nabla y\right) d x .
\end{gathered}
$$

Step 1. We apply the formula of integration by parts to the left-hand side of (3.2) keeping in mind that the initial assumptions on domain $\Omega$ and condition $\Delta y \in L^{2}(\Omega)$ imply $y \in$ $H^{2}(\Omega)$ ([13], Section 6.3). This yields

$$
\begin{aligned}
& \int_{\Omega}\left(\nabla y, \nabla\left(x-x_{0}, \nabla y\right)\right) d x+\int_{\Omega} y\left(x-x_{0}, \nabla y\right) d x \\
& =\sum_{i=1}^{N} \int_{\Omega} \frac{\partial y}{\partial x_{i}} \frac{\partial}{\partial x_{i}}\left[\sum_{j=1}^{N}\left(x_{j}-x_{0 j}\right) \frac{\partial y}{\partial x_{j}}\right] d x+\frac{1}{2} \int_{\Omega}\left(x-x_{0}, \nabla y^{2}\right) d x
\end{aligned}
$$




$$
\begin{aligned}
= & \sum_{i=1}^{N} \int_{\Omega}\left|\frac{\partial y}{\partial x_{i}}\right|^{2} d x+\sum_{i, j=1}^{N} \int_{\Omega}\left(x_{j}-x_{0 j}\right) \frac{\partial y}{\partial x_{i}} \frac{\partial^{2} y}{\partial x_{i} \partial x_{j}} d x \\
& +\frac{1}{2} \int_{\Omega}\left(x-x_{0}, \nabla y^{2}\right) d x \\
= & \int_{\Omega}|\nabla y|^{2} d x+\frac{1}{2} \sum_{i, j=1}^{N} \int_{\Omega}\left(x_{j}-x_{0 j}\right) \frac{\partial}{\partial x_{j}}\left(\frac{\partial y}{\partial x_{i}}\right)^{2} d x \\
& +\frac{1}{2} \sum_{j=1}^{N} \int_{\Omega}\left(x_{j}-x_{0 j}\right) \frac{\partial}{\partial x_{j}}\left(y^{2}\right) d x \\
= & \int_{\Omega}|\nabla y|^{2} d x+\frac{1}{2} \int_{\partial \Omega} \sum_{j=1}^{N}\left[\left(\sigma_{j}-x_{0 j}\right) v_{j}(\sigma)\right]\left[\sum_{i=1}^{N}\left(\frac{\partial y(\sigma)}{\partial \sigma_{i}}\right)^{2}\right] d \sigma \\
& -\frac{1}{2} \sum_{j=1}^{N} \int_{\Omega}\left[\sum_{i=1}^{N}\left(\frac{\partial y}{\partial x_{i}}\right)^{2}\right] d x+\frac{1}{2} \int_{\partial \Omega} \sum_{j=1}^{N}\left[\left(\sigma_{j}-x_{0 j}\right) v_{j}(\sigma)\right] y^{2}(\sigma) d \sigma-\frac{N}{2} \int_{\Omega} y^{2} d x \\
= & \left(1-\frac{N}{2}\right) \int_{\Omega}|\nabla y|^{2} d x-\frac{N}{2} \int_{\Omega} y^{2} d x \\
& +\frac{1}{2} \int_{\partial \Omega}\left(\sigma-x_{0}, v(\sigma)\right)|\nabla y(\sigma)|^{2} d \sigma+\frac{1}{2} \int_{\partial \Omega}\left(\sigma-x_{0}, v(\sigma)\right)|y(\sigma)|^{2} d \sigma .
\end{aligned}
$$

Since, by the star-shaped property of $\Omega$, we have $\left(\sigma-x_{0}, \nu(\sigma)\right) \geq 0$ for $\mathcal{H}^{N-1}$-a.a. $\sigma \in \partial \Omega$, it follows that

$$
\begin{gathered}
\int_{\Omega}\left(\nabla y, \nabla\left(x-x_{0}, \nabla y\right)\right) d x+\int_{\Omega} y\left(x-x_{0}, \nabla y\right) d x \\
\geq\left(1-\frac{N}{2}\right) \int_{\Omega}|\nabla y|^{2} d x-\frac{N}{2} \int_{\Omega}|y|^{2} d x .
\end{gathered}
$$

Step 2. Before proceeding, let us notice that the relation

$$
\int_{\Omega} f(y)(\nabla y, \psi) d x=\int_{\partial \Omega} F(y(\sigma))(v(\sigma), \psi(\sigma)) d \sigma-\int_{\Omega} F(y) \operatorname{div} \psi d x
$$

holds true for any vector-valued test function $\psi \in C^{1}(\bar{\Omega})^{N}$ provided $y \in H^{1}(\Omega)$ is a weak solution to (1.1)-(1.2). Indeed, let $T_{\varepsilon}: \mathbb{R} \rightarrow \mathbb{R}$ be the truncation operator defined in (2.11). By definition of $T_{\varepsilon}$, we have

$$
T_{\varepsilon}(y) \rightarrow y \quad \text { strongly in } H^{1}(\Omega) \text { and almost everywhere in } \Omega \text { as } \varepsilon \rightarrow 0 .
$$

Moreover, since $f(y) \in L^{2}(\Omega)$ and $f \in C_{\mathrm{loc}}(\mathbb{R})$, it follows from (3.5) that

$$
f\left(T_{\varepsilon}(y)\right) \rightarrow f(y) \quad \text { in } L^{2}(\Omega) \text { and almost everywhere in } \Omega .
$$

Then Lebesgue dominated theorem implies $f\left(T_{\varepsilon}(y)\right) \nabla T_{\varepsilon}(y) \rightarrow f(y) \nabla y$ in $L^{1}(\Omega)^{N}$. Taking into account the fact that

$$
f\left(T_{\varepsilon}(y)\right) \nabla T_{\varepsilon}(y)=\nabla F\left(T_{\varepsilon}(y)\right), \quad \forall \varepsilon>0,
$$


we conclude

$$
f(y) \nabla y=\nabla F(y) \quad \text { as elements of } L^{1}(\Omega) .
$$

As a result, the equality (3.4) is a direct consequence of the formula of integration by parts.

Now we are in a position to transform the right-hand side in (3.2). Indeed, due to equation (3.4), we have

$$
\begin{aligned}
& \int_{\Omega} f(y)\left(x-x_{0}, \nabla y\right) d x=\int_{\Omega}\left(x-x_{0}, \nabla F(y)\right) d x
\end{aligned}
$$

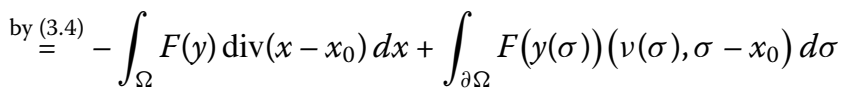

$$
\begin{aligned}
& \geq-N \int_{\Omega} F(y) d x
\end{aligned}
$$

because of the star-shaped property of $\Omega$ and the fact that $F(z) \geq F(0) \exp \left(C_{F}^{-1} z\right)$ over $\mathbb{R}$. Combining this inequality with (3.2) and (3.3), we arrive at the desired equation (3.1). The proof is complete.

Remark 3.2 In the following, we will use the following version of inequality (3.1):

$$
\int_{\Omega}|\nabla y|^{2} d x+\int_{\Omega}|y|^{2} d x \leq \frac{2 N}{N-2} \int_{\Omega} F(y) d x-\frac{2}{N-2} \int_{\Omega} g\left(x-x_{0}, \nabla y\right) d x .
$$

The next result is crucial in this section. Namely, we show that inequality (3.1) implies some prior estimate for the weak solutions $y \in Y$ to the original BVP.

Theorem 3.3 Let $y \in Y$ be a weak solution to BVP (1.1)-(1.2) such that $y$ satisfies the inequality (3.1). Then

$$
\begin{aligned}
& \int_{\Omega} y f(y) d x \leq C_{1}\|g\|_{L^{p}(\Omega)}^{2}+C_{2}\|g\|_{L^{p}(\Omega)}+C_{3}, \\
& \|y\|_{H^{1}(\Omega)} \leq C_{4}\|g\|_{L^{p}(\Omega)}+C_{5},
\end{aligned}
$$

for some positive constants $C_{i}, 1 \leq i \leq 5$, independent of $g$ and $y$.

Proof Combining the energy equality (2.6) with inequality (3.1), we get

$$
\begin{aligned}
& \left(\frac{N}{2}-1\right) \int_{\Omega} y f(y) d x+\left(\frac{N}{2}-1\right) \int_{\Omega} g y d x+\int_{\Omega} y^{2} d x \\
& \quad \leq N \int_{\Omega} F(y) d x-\int_{\Omega} g\left(x-x_{0}, \nabla y\right) d x .
\end{aligned}
$$

Hence, in view of estimate (2.7), we can rewrite the last relation as follows:

$$
\begin{aligned}
\int_{\Omega} y f(y) d x+\frac{2}{N-2} \int_{\Omega} y^{2} d x \leq & \frac{2 N}{N-2} \int_{\Omega} F(y) d x \\
& +|\Omega|^{\frac{p-2}{2 p}}\left(\frac{2 \operatorname{diam} \Omega}{N-2}+1\right)\|g\|_{L^{p}(\Omega)}\|y\|_{H^{1}(\Omega)} .
\end{aligned}
$$


For our further analysis, we set

$$
\Omega_{N}:=\left\{x \in \Omega: y(x)>\frac{4 N C_{F}}{N-2}\right\},
$$

where the constant $C_{F}$ is defined in (2.1). Since $F: \mathbb{R} \rightarrow(0,+\infty)$, it follows from (2.1) that

$$
\begin{aligned}
\frac{2 N}{N-2} \int_{\Omega} F(y) d x & \leq \frac{2 N C_{F}}{N-2} \int_{\Omega} f(y) d x \\
& \leq \frac{1}{2} \int_{\Omega_{N}} y f(y) d x+\frac{2 N C_{F}}{N-2} \int_{\Omega \backslash \Omega_{N}} f\left(\frac{4 N C_{F}}{N-2}\right) d x \\
& \leq \frac{1}{2} \int_{\Omega_{N}} y f(y) d x+\frac{2 N}{N-2} C_{F}|\Omega| f\left(\frac{4 N C_{F}}{N-2}\right)
\end{aligned}
$$

and

$$
\begin{aligned}
\int_{\Omega \backslash \Omega_{N}} y f(y) d x & \leq \frac{4 N C_{F}}{N-2} \int_{\Omega \backslash \Omega_{N}} f\left(\frac{4 N C_{F}}{N-2}\right) d x \\
& \leq \frac{4 N}{N-2} C_{F}|\Omega| f\left(\frac{4 N C_{F}}{N-2}\right) .
\end{aligned}
$$

Then inequality (3.9) yields the following relation:

$$
\begin{aligned}
& \int_{\Omega_{N}} y f(y) d x+\frac{2}{N-2} \int_{\Omega} y^{2} d x \\
& =\int_{\Omega} y f(y) d x-\int_{\Omega \backslash \Omega_{N}} y f(y) d x+\frac{2}{N-2} \int_{\Omega} y^{2} d x \\
& \quad \stackrel{\text { by (3.9) }}{\leq} \frac{1}{2} \int_{\Omega_{N}} y f(y) d x+\frac{2 N}{N-2} C_{F}|\Omega| f\left(\frac{4 N C_{F}}{N-2}\right) \\
& \quad-\int_{\Omega \backslash \Omega_{N}} y f(y) d x+|\Omega|^{\frac{p-2}{2 p}}\left(\frac{2 \operatorname{diam} \Omega}{N-2}+1\right)\|g\|_{L^{p}(\Omega)}\|y\|_{H^{1}(\Omega)} .
\end{aligned}
$$

Therefore,

$$
\begin{aligned}
\frac{1}{2} \int_{\Omega} y f(y) d x \leq & \frac{2 N}{N-2} C_{F}|\Omega| f\left(\frac{4 N C_{F}}{N-2}\right)-\frac{1}{2} \int_{\Omega \backslash \Omega_{N}} y f(y) d x \\
& +|\Omega|^{\frac{p-2}{2 p}}\left(\frac{2 \operatorname{diam} \Omega}{N-2}+1\right)\|g\|_{L^{p}(\Omega)}\|y\|_{H^{1}(\Omega)} .
\end{aligned}
$$

As a result, we get from (3.10) and the previous inequality

$$
\begin{aligned}
\int_{\Omega} y f(y) d x & \leq \frac{8 N}{N-2} C_{F}|\Omega| f\left(\frac{4 N C_{F}}{N-2}\right)+2|\Omega|^{\frac{p-2}{2 p}}\left(\frac{2 \operatorname{diam} \Omega}{N-2}+1\right)\|g\|_{L^{p}(\Omega)}\|y\|_{H^{1}(\Omega)} \\
& =\widehat{C}_{1}+2 \widehat{C}_{2}\|g\|_{L^{p}(\Omega)}\|y\|_{H^{1}(\Omega)} .
\end{aligned}
$$

Finally using the energy equality (2.6), we obtain

$$
\|y\|_{H^{1}(\Omega)}^{2} \leq \widehat{C}_{1}+3 \widehat{C}_{2}\|g\|_{L^{p}(\Omega)}\|y\|_{H^{1}(\Omega)}
$$


and this implies the desired estimate (3.8). In order to establish the estimate (3.7), it is enough to make use of (3.8) in (3.11). The proof is complete.

Remark 3.4 It is worth to notice that inequality (3.1) makes sense even if we do not assume the fulfillment of inclusion $\Delta y \in L^{2}(\Omega)$ but only have $y \in Y$ and $g \in L^{p}(\Omega)$. At the same time it is unknown whether this inequality holds for an arbitrary weak solution to BVP (1.1)-(1.2). Since the existence and uniqueness of the weak solutions to the original BVP is an open question for arbitrary given distribution $g \in L^{p}(\Omega)$ with $p>2$, the following result reflects some interesting properties of weak solutions satisfying inequality (3.1).

Proposition 3.5 Let $(g, y)$ be a given pair in $L^{p}(\Omega) \times H^{1}(\Omega)$ with $p>2$. Let $\left\{\left(g_{k}, y_{k}\right)\right\}_{k \in \mathbb{N}} \subset$ $L^{p}(\Omega) \times Y$ be a sequence such that, for each $k \in \mathbb{N}$, the pairs $\left(g_{k}, y_{k}\right)$ are related by the integral identity (2.3), satisfy inequality (3.1), and

$$
\left(g_{k}, y_{k}\right) \rightarrow(g, y) \quad \text { weakly in } L^{p}(\Omega) \times H^{1}(\Omega) \text { as } k \rightarrow \infty .
$$

Then $y$ is a weak solution to BVP (1.1)-(1.2) for the given $g \in L^{p}(\Omega)$, the pair $(g, y)$ satisfies the inequality (3.1), and

$$
f\left(y_{k}\right) \rightarrow f(y) \quad \text { in } L^{1}(\Omega) \text { as } k \rightarrow \infty .
$$

Proof By the Rellich-Kondrachov theorem, the embedding $H^{1}(\Omega) \hookrightarrow L^{2}(\Omega)$ is compact. Hence, the weak convergence $y_{k} \rightarrow y$ in $H^{1}(\Omega)$ implies strong convergence in $L^{2}(\Omega)$. Therefore, up to a subsequence, we can suppose that $y_{k}(x) \rightarrow y(x)$ for almost every point $x \in \Omega$. As a result, we have the pointwise convergence: $f\left(y_{k}\right) \rightarrow f(y)$ everywhere in $\Omega$. Let us show that this implies the strong convergence (3.13).

With that in mind we recall that a sequence $\left\{f_{k}\right\}_{k \in \mathbb{N}}$ is called equi-integrable on $\Omega$ if for any $\delta>0$, there is a $\tau=\tau(\delta)$ such that $\int_{S}\left|f_{k}\right| d x<\delta$ for every measurable subset $S \subset \Omega$ of Lebesgue measure $|S|<\tau$. Let us show that the sequence $\left\{f\left(y_{k}\right)\right\}_{k \in \mathbb{N}}$ is equi-integrable on $\Omega$. To do so, we take $m>0$ such that

$$
m>\frac{2\left(C_{1} \sup _{k \in \mathbb{N}}\left\|g_{k}\right\|_{L^{p}(\Omega)}^{2}+C_{2} \sup _{k \in \mathbb{N}}\left\|g_{k}\right\|_{L^{p}(\Omega)}+C_{3}\right)}{\delta},
$$

where the constants $C_{i}, i=1,2,3$, are as in (3.7). We also set $\tau=\delta /(2 f(m))$. Then, for every measurable set $S \subset \Omega$ with $|S|<\tau$, we have

$$
\begin{aligned}
\int_{S} f\left(y_{k}\right) d x & \leq \int_{\left\{x \in S: y_{k}(x)>m\right\}} f\left(y_{k}\right) d x+\int_{\left\{x \in S: y_{k}(x) \leq m\right\}} f\left(y_{k}\right) d x \\
& \leq \frac{1}{m} \int_{\left\{x \in S: y_{k}(x)>m\right\}} y_{k} f\left(y_{k}\right) d x+\int_{\left\{x \in S: y_{k}(x) \leq m\right\}} f(m) d x \\
& \stackrel{\text { by (3.7) }}{\leq} \frac{C_{1}\left\|g_{k}\right\|_{L^{p}(\Omega)}^{2}+C_{2}\left\|g_{k}\right\|_{L^{p}(\Omega)}+C_{3}}{m}+f(m)|S| \\
& \stackrel{\text { by (3.14) } \delta}{\leq} \frac{\delta}{2}+\frac{\delta}{2} .
\end{aligned}
$$

As a result, the assertion (3.13) is a direct consequence of Lebesgue's convergence theorem. Hence, $y \in Y$ and it is easy to show that the limit pair $(g, y)$ is related by the integral identity (2.3). Indeed, in view of the initial assumptions and the strong convergence property (3.13), 
the limit passage in

$$
\int_{\Omega}\left(\nabla y_{k}, \nabla \varphi\right) d x+\int_{\Omega} y_{k} \varphi d x=\int_{\Omega} f\left(y_{k}\right) \varphi d x+\int_{\Omega} g_{k} \varphi d x, \quad \forall \varphi \in C_{0}^{\infty}\left(\mathbb{R}^{N}\right),
$$

becomes trivial. Thus, $y$ is a weak solution to BVP (1.1)-(1.2) for the given $g \in L^{p}(\Omega)$.

Our next aim is to prove that $(g, y)$ satisfies (3.1). With that in mind we make use of the following result (see Boccardo and Murat [14]): if $y_{k} \rightarrow y$ in $H^{1}(\Omega)$ and the sequence $\left\{\Delta y_{k}\right\}_{k \in \mathbb{N}}$ is bounded in $L^{1}(\Omega)$, then, within a subsequence, $\nabla y_{k}(x) \rightarrow \nabla y(x)$ almost everywhere as $k \rightarrow \infty$. Indeed, as follows from (2.3),

$$
\int_{\Omega}\left|\Delta y_{k}\right| d x \leq \int_{\Omega}\left|y_{k}\right| d x+\int_{\Omega}\left|f\left(y_{k}\right)\right| d x+\int_{\Omega}\left|g_{k}\right| d x .
$$

Hence, in view of (3.15) and $L^{p}(\Omega)$-boundedness of $\left\{g_{k}\right\}_{k \in \mathbb{N}}$ with $p>2$, we have

$$
\sup _{k \in \mathbb{N}}\left\|\Delta y_{k}\right\|_{L^{1}(\Omega)}<+\infty
$$

Thus, in the following we may suppose that

$$
\nabla y_{k} \rightarrow \nabla y \quad \text { in } L^{2}(\Omega)^{N} \quad \text { and } \quad \nabla y_{k}(x) \rightarrow \nabla y(x) \quad \text { a.e. in } \Omega \text {. }
$$

Let us show that this fact implies the strong convergence of gradients $\nabla y_{k} \rightarrow \nabla y$ in $L^{p^{\prime}}(\Omega)^{N}$ with $p^{\prime}=p /(p-1)$. Indeed, for an arbitrary small set $A$, by the Hölder inequality for any $q, q^{\prime} \geq 1$ such that $1 / q+1 / q^{\prime}=1$, we have

$$
\int_{A}\left|\nabla y_{k}-\nabla y\right|^{p^{\prime}} d x \leq\left(\int_{A}\left|\nabla y_{k}-\nabla y\right|^{p^{\prime} q} d x\right)^{1 / q}\left(\int_{A} 1^{q^{\prime}} d x\right)^{1 / q^{\prime}} .
$$

Having chosen $q>2$ such that $p^{\prime} q=2$, we obtain

$$
\frac{1}{q^{\prime}}=1-\frac{1}{q}=1-\frac{p^{\prime}}{2}=\frac{2-p^{\prime}}{2}=\frac{p-2}{2(p-1)} \quad \text { or } \quad q^{\prime}=\frac{2(p-1)}{p-2} .
$$

Then

$$
\int_{A}\left|\nabla y_{k}-\nabla y\right|^{p^{\prime}} d x \leq|A|^{\frac{p-2}{2(p-1)}} \sup _{k \in \mathbb{N}}\left\|\nabla y_{k}-\nabla y\right\|_{L^{2}(\Omega)^{N}}^{p^{\prime}} \leq C|A|^{\frac{p-2}{2(p-1)}},
$$

that is, the sequence $\left\{\left|\nabla y_{k}-\nabla y\right|^{p^{\prime}}\right\}_{k \in \mathbb{N}}$ is equi-integrable. Combining this fact with (3.16), by the Lebesgue convergence theorem, we conclude: $\left|\nabla y_{k}-\nabla y\right|^{p^{\prime}} \rightarrow 0$ strongly in $L^{1}(\Omega)$, and, therefore,

$$
\nabla y_{k} \rightarrow \nabla y \quad \text { strongly in } L^{p^{\prime}}(\Omega)^{N} \text { with } p^{\prime}=p /(1-p) .
$$

As a result, we get

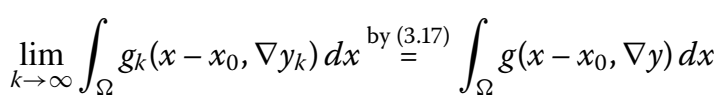

(as a product of weakly and strongly convergent sequences), 


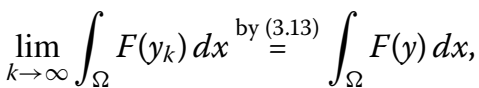

$$
\begin{aligned}
& \liminf _{k \rightarrow \infty} \int_{\Omega}|\nabla y|^{2} d x \stackrel{\text { by (3.12) }}{\geq} \int_{\Omega}|\nabla y|^{2} d x .
\end{aligned}
$$

Then we can pass to the limit in the inequality (3.1) to finally obtain

$$
\begin{aligned}
\left(\frac{N}{2}-1\right) \int_{\Omega}|\nabla y|^{2} d x+\frac{N}{2} \int_{\Omega}|y|^{2} d x \leq & \left(\frac{N}{2}-1\right) \liminf _{k \rightarrow \infty} \int_{\Omega}\left|\nabla y_{k}\right|^{2} d x \\
& +\frac{N}{2} \liminf _{k \rightarrow \infty} \int_{\Omega}\left|y_{k}\right|^{2} d x \\
\leq & \liminf _{k \rightarrow \infty}\left[N \int_{\Omega} F\left(y_{k}\right) d x-\int_{\Omega} g_{k}\left(x-x_{0}, \nabla y_{k}\right) d x\right] \\
= & N \int_{\Omega} F(y) d x-\int_{\Omega} g\left(x-x_{0}, \nabla y\right) d x .
\end{aligned}
$$

The proof is complete.

\section{Fictitious controls and associated optimal control problems}

Let us consider the following sequence of optimal control problems (OCPs) associated with BVP (1.1)-(1.2):

$$
\left\{\left(\inf _{(u, y) \in \Xi_{\Delta}} J_{\varepsilon}(u, y)\right), \varepsilon \rightarrow 0\right\}
$$

where

$$
\left.\begin{array}{l}
\Xi_{\Delta}=\left\{(u, y) \mid \begin{array}{l}
u \in L^{p}(\Omega), \quad y \in Y, \\
\int_{\Omega}[(\nabla y, \nabla \varphi)+y \varphi] d x \\
=\int_{\Omega} f(y) \varphi d x+\int_{\Omega} u \varphi d x, \quad \forall \varphi \in C_{0}^{\infty}\left(\mathbb{R}^{N}\right), \\
J_{\varepsilon}(u, y)<+\infty
\end{array}\right.
\end{array}\right\},
$$

Here, we consider the function $u \in L^{p}(\Omega)$ as a fictitious control and $\varepsilon$ is a small parameter. Hereinafter we assume that the parameter $\varepsilon$ varies within a strictly decreasing sequence of positive real numbers which converge to zero.

It is worth to notice that, in contrast to the original BVP (1.1)-(1.2) for which it is unknown whether the set of its weak solutions is non-empty, each of the parametrized OCPs (4.1)-(4.3) is regular in the following sense: the set of feasible solutions $\Xi_{\Delta}$ is always nonempty. Indeed, it is enough to take an arbitrary function $\tilde{y} \in C^{\infty}(\bar{\Omega})$ with $\frac{\partial y}{\partial v}=0$ on $\partial \Omega$ and to put $\tilde{u}:=-\Delta \widetilde{y}+\tilde{y}-f(\tilde{y})$. Since $\tilde{y} \in Y$ and $\Delta \widetilde{y} \in L^{2}(\Omega)$, it follows from Proposition 3.1 and Definition 2.1 that $J_{\varepsilon}(\widetilde{u}, \widetilde{y})<+\infty$ and the pair $(\widetilde{u}, \widetilde{y})$ is related by integral identity (2.3). Hence, $(\widetilde{u}, \widetilde{y}) \in \Xi_{\Delta}$.

Let us show that the OCPs (4.1)-(4.3) are solvable for each $\varepsilon>0$. 
Theorem 4.1 Let $g \in L^{p}(\Omega)$ be a given distribution. Then for every $\varepsilon>0$ there exists at least one pair $\left(u_{\varepsilon}^{0}, y_{\varepsilon}^{0}\right) \in \Xi_{\Delta}$ such that

$$
J_{\varepsilon}\left(u_{\varepsilon}^{0}, y_{\varepsilon}^{0}\right)=\inf _{(u, y) \in \Xi_{\Delta}} J_{\varepsilon}(u, y) .
$$

Proof Since $\Xi_{\Delta} \neq \emptyset$, it follows that for given $\varepsilon>0$ and $g \in L^{p}(\Omega)$ there exists a minimizing sequence $\left\{\left(u_{\varepsilon, k}, y_{\varepsilon, k}\right)\right\}_{k \in \mathbb{N}}$ to OCP (4.1)-(4.3), i.e.

$$
\begin{aligned}
\lim _{k \rightarrow \infty} J_{\varepsilon}\left(u_{\varepsilon, k}, y_{\varepsilon, k}\right) & =\inf _{(u, y) \in \Xi_{\varepsilon}} J_{\varepsilon}(u, y) \leq J_{\varepsilon}(\widetilde{u}, \widetilde{y}) \\
& \leq\|\Delta \widetilde{y}\|_{L^{2}(\Omega)}^{2}+\|g-\widetilde{u}\|_{L^{p}(\Omega)}^{p}=\widetilde{C}<+\infty .
\end{aligned}
$$

As a result, we have

$$
\sup _{k \in N}\left\|\Delta y_{\varepsilon, k}\right\|_{L^{2}(\Omega)}^{2} \stackrel{\text { by }}{\leq} \stackrel{(4.4)}{\leq} \varepsilon^{-1} \widetilde{C}
$$

and this estimate implies that each of the pairs $\left(u_{\varepsilon, k}, y_{\varepsilon, k}\right)$ satisfies the inequality (see (3.6) and Proposition 3.1)

$$
\begin{aligned}
\int_{\Omega}\left|\nabla y_{\varepsilon, k}\right|^{2} d x+\int_{\Omega}\left|y_{\varepsilon, k}\right|^{2} d x \leq & \frac{2 N}{N-2} \int_{\Omega} F\left(y_{\varepsilon, k}\right) d x \\
& -\frac{2}{N-2} \int_{\Omega} u_{\varepsilon, k}\left(x-x_{0}, \nabla y_{\varepsilon, k}\right) d x .
\end{aligned}
$$

Moreover, in view of Theorem 3.3, we have

$$
\begin{aligned}
& \sup _{k \in N}\left[\left\|y_{\varepsilon, k}\right\|_{H^{1}(\Omega)}^{2}+\left\|u_{\varepsilon, k}\right\|_{L^{p}(\Omega)}^{p}\right] \\
& \quad \stackrel{\text { by (4.5) and (3.8) }}{\leq} \sup _{k \in N}\left[\left(C_{4}\left\|u_{\varepsilon, k}\right\|_{L^{p}(\Omega)}+C_{5}\right)^{2}+\left\|u_{\varepsilon, k}\right\|_{L^{p}(\Omega)}^{p}\right] \\
& \quad \leq 2^{p-1} C_{5}^{p}+p\left(1+2^{p-1} C_{4}^{p}\right) \sup _{k \in N} J_{\varepsilon}\left(u_{\varepsilon, k}, y_{\varepsilon, k}\right) \stackrel{\text { by }}{<} \stackrel{(4.4)}{<}+\infty
\end{aligned}
$$

for $\varepsilon>0$ small enough, where the constants $C_{4}$ and $C_{5}$ do not depend on $\varepsilon$.

Thus, passing to subsequences, if necessary, we can suppose that there exists a pair $\left(u_{\varepsilon}^{0}, y_{\varepsilon}^{0}\right) \in L^{p}(\Omega) \times H^{1}(\Omega)$ such that

$$
u_{\varepsilon, k} \rightarrow u_{\varepsilon}^{0} \quad \text { in } L^{p}(\Omega) \quad \text { and } \quad y_{\varepsilon, k} \rightarrow y_{\varepsilon}^{0} \quad \text { in } H^{1}(\Omega) \text { as } k \rightarrow \infty .
$$

Hence, in view of Proposition 3.5, $y_{\varepsilon}^{0}$ is a weak solution to BVP

$$
\begin{aligned}
& -\Delta y+y=f(y)+u_{\varepsilon}^{0} \quad \text { in } \Omega, \\
& \frac{\partial y}{\partial v}=0 \quad \text { on } \partial \Omega,
\end{aligned}
$$

the pair $\left(u_{\varepsilon}^{0}, y_{\varepsilon}^{0}\right)$ satisfies the inequality (4.6), and

$$
f\left(y_{\varepsilon, k}\right) \rightarrow f\left(y_{\varepsilon}^{0}\right) \quad \text { in } L^{1}(\Omega) \text { as } k \rightarrow \infty .
$$


Thus, $\left(u_{\varepsilon}^{0}, y_{\varepsilon}^{0}\right) \in \Xi$, where

$$
\Xi=\left\{(u, y) \mid \begin{array}{l}
u \in L^{p}(\Omega), \quad y \in Y, \\
\int_{\Omega}[(\nabla y, \nabla \varphi)+y \varphi] d x \\
=\int_{\Omega} f(y) \varphi d x+\int_{\Omega} u \varphi d x, \quad \forall \varphi \in C_{0}^{\infty}\left(\mathbb{R}^{N}\right), \\
\int_{\Omega}\left[|\nabla y|^{2}+y^{2}\right] d x \\
\leq \frac{2 N}{N-2} \int_{\Omega} F(y) d x-\frac{2}{N-2} \int_{\Omega} u\left(x-x_{0}, \nabla y\right) d x
\end{array}\right\} .
$$

It remains to show that $\left(u_{\varepsilon}^{0}, y_{\varepsilon}^{0}\right) \in \Xi_{\Delta}$ and $\left(u_{\varepsilon}^{0}, y_{\varepsilon}^{0}\right)$ is an optimal pair to the constrained minimization problem (4.1) for a given $\varepsilon>0$. Taking into account the estimates (4.5) and (4.7), it is easy to see that the sequence $\left\{y_{\varepsilon, k}\right\}_{k \in \mathbb{N}}$ is bounded in the Banach space $H_{\Delta}^{1}(\Omega)$ connected with the Laplace operator by the formula (see, for instance, [15])

$$
H_{\Delta}^{1}(\Omega)=\left\{y \in H^{1}(\Omega): \Delta y \in L^{2}(\Omega)\right\}
$$

where the norm in $H_{\Delta}^{1}(\Omega)$ can be defined in the standard way as the norm of the graph:

$$
\|y\|_{H_{\Delta}^{1}(\Omega)}^{2}=\|y\|_{H^{1}(\Omega)}^{2}+\|\Delta y\|_{L^{2}(\Omega)}^{2} .
$$

Hence, the limit properties (4.8) can be supplemented by the following one:

$$
\Delta y_{\varepsilon, k} \rightarrow \Delta y_{\varepsilon}^{0} \quad \text { in } L^{2}(\Omega) \text { as } k \rightarrow \infty .
$$

As a result, making use of the lower semi-continuity property of the cost functional $J_{\varepsilon}: L^{p}(\Omega) \times H^{1}(\Omega) \rightarrow \mathbb{R}$ with respect to the weak convergence in $L^{p}(\Omega) \times H_{\Delta}^{1}(\Omega)$, we arrive at the following relation:

$$
\inf _{(u, y) \in \Xi_{\Delta}} J_{\varepsilon}(u, y)=\lim _{k \rightarrow \infty} J_{\varepsilon}\left(u_{\varepsilon, k}, y_{\varepsilon, k}\right) \geq J_{\varepsilon}\left(u_{\varepsilon}^{0}, y_{\varepsilon}^{0}\right) .
$$

Hence, $J_{\varepsilon}\left(u_{\varepsilon}^{0}, y_{\varepsilon}^{0}\right)<+\infty$ (which implies $\left.\left(u_{\varepsilon}^{0}, y_{\varepsilon}^{0}\right) \in \Xi_{\Delta}\right)$ and $\left(u_{\varepsilon}^{0}, y_{\varepsilon}^{0}\right)$ is an optimal pair to the corresponding optimization problem (4.1).

Our next intention is to discuss the uniqueness property of the solutions to OCPs (4.1). We begin with the following noteworthy characteristic of the set $\Xi_{\Delta}$.

Lemma 4.2 Assume that, in addition to the property (2.1), the function $F \in C_{\mathrm{loc}}^{1}(\mathbb{R})$ is such that its derivative $f=F^{\prime}: \mathbb{R} \rightarrow(0, \infty)$ is a strictly convex function. Then the set

$$
\Lambda=\left\{u \in L^{p}(\Omega): \exists y \in Y \text { such that }(u, y) \in \mathbf{\Xi}_{\Delta}\right\}
$$

is non-empty and convex. 
Proof Since the set of feasible solution $\Xi_{\Delta}$ is non-empty, it follows from (4.11) that $\Lambda \neq \emptyset$. Let us establish the convexity of $\Lambda$. Let $\left(u_{1}, y_{1}\right)$ and $\left(u_{2}, y_{2}\right)$ be two different pairs of $\Xi_{\Delta}$. It is clear that in this case we have $y_{1} \neq y_{2}$ and $u_{1}, u_{2} \in \Lambda$. Let $\lambda \in(0,1)$. We set

$$
u=\lambda u_{1}+(1-\lambda) u_{2}, \quad y=\lambda y_{1}+(1-\lambda) y_{2} .
$$

Our aim is to show that $u \in \Lambda$.

Since for Lipschitz $\Omega$ the mapping $(-\Delta+I): H^{1}(\Omega) \rightarrow\left(H^{1}(\Omega)\right)^{*}$ is an isomorphism, we can define, in a unique way, a distribution $z \in H^{1}(\Omega)$ as follows:

$$
-\Delta z+z=u=\lambda u_{1}+(1-\lambda) u_{2} \quad \text { in } \Omega \quad \text { and } \quad \frac{\partial z}{\partial v}=0 \quad \text { on } \partial \Omega
$$

By the initial assumptions, we have

$$
-\Delta y_{1}+y_{1}=f\left(y_{1}\right)+u_{1} \quad \text { and } \quad-\Delta y_{2}+y_{2}=f\left(y_{2}\right)+u_{2} .
$$

Hence,

$$
-\Delta y+y=\lambda f\left(y_{1}\right)+(1-\lambda) f\left(y_{2}\right)+u \quad \text { in } \Omega \quad \text { and } \quad \frac{\partial y}{\partial v}=0 \quad \text { on } \partial \Omega .
$$

Taking into account the facts that $f=F^{\prime}(z)>0$ almost everywhere in $\Omega$ (see (2.1)) and $f$ satisfies the Jensen inequality

$$
f(y) \leq \lambda f\left(y_{1}\right)+(1-\lambda) f\left(y_{2}\right)
$$

we obtain

$$
-\Delta z+z \leq f(z)+u \text { and }-\Delta y+y \stackrel{\text { by (4.12) and (4.13) }}{\geq} F^{\prime}(y)+u,
$$

i.e. $z$ is a subsolution to the boundary value problem

$$
-\Delta \psi+\psi=f(\psi)+u \quad \text { in } \Omega, \quad \frac{\partial \psi}{\partial v}=0 \quad \text { on } \partial \Omega
$$

and $y$ is its supersolution. Moreover, since

$$
-\Delta y+y \geq f(y)+u \stackrel{\text { by }(2.1)}{\geq} u=-\Delta z+z \quad \text { in } \Omega
$$

it follows that $-\Delta(y-z)+(y-z) \geq 0$. Hence, by the strong maximum principle [16] (see also Chapter 2 in [17]), we conclude that $y \geq z$ in $\Omega$. Thus, following the classical techniques introduced by Sattinger [18], we deduce that for a given control $u=$ $\lambda u_{1}+(1-\lambda) u_{2}$ there exists a solution $\psi$ to the above boundary value problem such that

$$
z(x) \leq \psi(x) \leq y(x) \quad \text { almost everywhere in } \Omega .
$$


Moreover, as follows from (4.16) and the fact that $z, y \in H^{1}(\Omega)$, we have $\psi \in H^{1}(\Omega)$ and

$$
\begin{aligned}
\int_{\Omega} f(\psi) d x & \stackrel{\text { by (4.16) and (2.1) }}{\leq} \int_{\Omega} f(y) d x \\
& \stackrel{\text { by (4.13) }}{\leq} \lambda\left\|f\left(y_{1}\right)\right\|_{L^{1}(\Omega)}+(1-\lambda)\left\|f\left(y_{2}\right)\right\|_{L^{1}(\Omega)}<+\infty .
\end{aligned}
$$

Hence, $\psi \in Y$ and, therefore, $\psi$ is a weak solution to BVP (4.15).

In order to prove the inclusion $u \in \Lambda$, it remains to establish that $(u, \psi) \in \Xi_{\Delta}$. To do so, we note that

$$
\begin{aligned}
-\Delta z+z & =u \leq \underbrace{f(\psi)+u}_{-\Delta \psi+\psi} \stackrel{\text { by }}{\stackrel{(4.16)}{\leq}} f(y)+u \\
& \stackrel{\text { by (4.13) }}{\leq} \lambda\left(f\left(y_{1}\right)+u_{1}\right)+(1-\lambda)\left(f\left(y_{2}\right)+u_{2}\right) \\
& =\lambda\left(-\Delta y_{1}+y_{1}\right)+(1-\lambda)\left(-\Delta y_{2}+y_{2}\right) .
\end{aligned}
$$

Since $\Delta z \in L^{2}(\Omega)$ and $y_{i} \in H_{\Delta}^{1}(\Omega), i=1,2$, it follows from (4.17) that $\Delta \psi \in L^{2}(\Omega)$. Hence, $J_{\varepsilon}(u, \psi)<+\infty$. So, $(u, \psi)$ is an admissible pair to each of OCPs (4.1). Thus, $u \in \Lambda$.

Remark 4.3 In general, we cannot assert that the set $\Lambda$ is closed in $L^{p}(\Omega)$. Indeed, let $\left\{u_{k}\right\}_{k \in \mathbb{N}} \subset \Lambda$ be a sequence such that $u_{k} \rightarrow u$ in $L^{p}(\Omega)$ as $k \rightarrow \infty$. Let $\left\{y_{k}\right\}_{k \in \mathbb{N}} \in Y$ be the corresponding sequence of states, that is, $\left(u_{k}, y_{k}\right) \in \Xi_{\Delta}$ for each $k \in \mathbb{N}$. Then, in view of estimate (3.8), we can suppose that there exists a distribution $y \in H^{1}(\Omega)$ such that $y_{k} \rightarrow$ $y$ in $H^{1}(\Omega)$. Hence, by Proposition 3.5, we have: $y$ is a weak solution to BVP (1.1)-(1.2) with $g=u$ and the pair $(u, y)$ satisfies the inequality (3.1). However, it is unknown whether $J_{\varepsilon}(u, y)<+\infty$, because the given choice of the sequence of feasible pairs $\left\{\left(u_{k}, y_{k}\right)\right\}_{k \in \mathbb{N}} \subset \Xi_{\Delta}$ does not guarantee the $L^{2}(\Omega)$-boundedness of the corresponding Laplacians $\left\{\Delta y_{k}\right\}_{k \in \mathbb{N}}$. So, it may happen that $\Delta y \notin L^{2}(\Omega)$. At the same time, as follows from Theorem 4.1, the lack of $L^{p}(\Omega)$-closedness of $\Lambda$ is not a restrictive option for the solvability of the corresponding OCP. Moreover, as we show in the next assertion, the main result of Theorem 4.1 can be essentially specified.

Theorem 4.4 Under assumptions of Lemma 4.2, each of the OCPs (4.1) has a unique solution.

Proof Let us assume the converse. Namely, let $\varepsilon>0$ be a fixed value and let $\left(u_{\varepsilon, 1}, y_{\varepsilon, 1}\right)$ and $\left(u_{\varepsilon, 2}, y_{\varepsilon, 2}\right)$ be two different pairs such that $\left(u_{\varepsilon, 1}, y_{\varepsilon, 1}\right) \neq\left(u_{\varepsilon, 2}, y_{\varepsilon, 2}\right)$ and

$$
\left(u_{\varepsilon, 1}, y_{\varepsilon, 1}\right),\left(u_{\varepsilon, 2}, y_{\varepsilon, 2}\right) \in \Xi_{\Delta}, J_{\varepsilon}\left(u_{\varepsilon, 1}, y_{\varepsilon, 1}\right)=J_{\varepsilon}\left(u_{\varepsilon, 2}, y_{\varepsilon, 2}\right)=\inf _{(u, y) \in \Xi_{\Delta}} J_{\varepsilon}(u, y)
$$

We set $u_{\varepsilon}=\left(u_{\varepsilon, 1}+u_{\varepsilon, 2}\right) / 2$. By analogy with the proof of Lemma 4.2, it is easy to show that there exists a distribution $\psi_{\varepsilon} \in H^{1}(\Omega)$ such that $\left(u_{\varepsilon}, \psi_{\varepsilon}\right) \in \mathbf{\Xi}_{\Delta}$ and

$$
\psi_{\varepsilon} \leq\left(y_{\varepsilon, 1}+y_{\varepsilon, 2}\right) / 2 \quad \text { a.e. in } \Omega
$$


In fact, because of the strict convexity of $f$, it can be shown that the previous inequality is strict in $\Omega$. Indeed, since

$$
\begin{aligned}
-\Delta & \left(\frac{1}{2} y_{\varepsilon, 1}+\frac{1}{2} y_{\varepsilon, 2}-\psi_{\varepsilon}\right)+\left(\frac{1}{2} y_{\varepsilon, 1}+\frac{1}{2} y_{\varepsilon, 2}-\psi_{\varepsilon}\right) \\
& =\frac{1}{2}\left(f\left(y_{\varepsilon, 1}\right)+u_{\varepsilon, 1}\right)+\frac{1}{2}\left(f\left(y_{\varepsilon, 2}\right)+u_{\varepsilon, 2}\right)-f\left(\psi_{\varepsilon}\right)-u_{\varepsilon} \\
& =\frac{1}{2}\left(f\left(y_{\varepsilon, 1}\right)+f\left(y_{\varepsilon, 2}\right)\right)-f\left(\psi_{\varepsilon}\right) \\
& \geq \frac{1}{2}\left(f\left(y_{\varepsilon, 1}\right)+f\left(y_{\varepsilon, 2}\right)\right)-f\left(\frac{y_{\varepsilon, 1}+y_{\varepsilon, 2}}{2}\right) \geq 0
\end{aligned}
$$

by the Jensen inequality, and $\frac{1}{2}\left(f\left(y_{\varepsilon, 1}\right)+f\left(y_{\varepsilon, 2}\right)\right)-f\left(\frac{y_{\varepsilon, 1}+y_{\varepsilon, 2}}{2}\right) \neq 0$ on $\Omega$ because of the strict convexity of $f$, it follows that $\left(y_{\varepsilon, 1}+y_{\varepsilon, 2}\right) / 2>\psi_{\varepsilon}$ in $\Omega$ by the strong maximum principle [16, 17]. As a result, we obtain an inequality

$$
\begin{aligned}
J_{\varepsilon}\left(u_{\varepsilon}, \psi_{\varepsilon}\right)= & \frac{\varepsilon}{2} \int_{\Omega}\left|\Delta \psi_{\varepsilon}\right|^{2} d x+\frac{1}{p} \int_{\Omega}\left|g-u_{\varepsilon}\right|^{p} d x \\
\leq & \frac{\varepsilon}{8} \int_{\Omega}\left|\Delta y_{\varepsilon, 1}+\Delta y_{\varepsilon, 2}\right|^{2} d x+\frac{1}{p 2^{p}} \int_{\Omega}\left|g-u_{\varepsilon, 1}+g-u_{\varepsilon, 2}\right|^{p} d x \\
< & \frac{\varepsilon}{4} \int_{\Omega}\left|\Delta y_{\varepsilon, 1}\right|^{2} d x+\frac{1}{2 p} \int_{\Omega}\left|g-u_{\varepsilon, 1}\right|^{p} d x \\
& +\frac{\varepsilon}{4} \int_{\Omega}\left|\Delta y_{\varepsilon, 2}\right|^{2} d x+\frac{1}{2 p} \int_{\Omega}\left|g-u_{\varepsilon, 2}\right|^{p} d x \\
= & \frac{1}{2} J_{\varepsilon}\left(u_{\varepsilon, 1}, y_{\varepsilon, 1}\right)+\frac{1}{2} J_{\varepsilon}\left(u_{\varepsilon, 2}, y_{\varepsilon, 2}\right)=\inf _{(u, y) \in \Xi_{\varepsilon}} J_{\varepsilon}(u, y),
\end{aligned}
$$

which is a contradiction with the fact that $\left(u_{\varepsilon}, \psi_{\varepsilon}\right)$ is an admissible pair to the problem (4.1).

Remark 4.5 As was mentioned in Remark 4.3, the convex set $\Lambda$ is not closed in $L^{p}(\Omega)$. Let $\bar{\Lambda} \subset L^{p}(\Omega)$ denote the closure of $\Lambda$ with respect to the strong topology of $L^{p}(\Omega)$. Then by Mazur's theorem this set coincides with the sequential weak closure of $\Lambda$ in $L^{p}(\Omega)$, that is, $\bar{\Lambda}=\mathrm{cl}_{w-L^{p}(\Omega)} \Lambda$.

\section{Variational properties of fictitious optimal control problems}

Before setting foot in the asymptotic analysis of the sequence of OCPs (4.1) as $\varepsilon \rightarrow 0$, we define the $\mu$-topology on $L^{p}(\Omega) \times H^{1}(\Omega)$ as the product of weak topologies of $L^{p}(\Omega)$ and $H^{1}(\Omega)$, respectively. Let $\mathrm{cl}_{\mu} \Xi_{\Delta}$ be the sequential closure of the set of feasible pairs $\Xi_{\Delta}$ with respect to the $\mu$-topology. In view of Proposition 3.5, it is clear that in this case we have

$$
\bar{\Lambda}=\left\{u \in L^{p}(\Omega): \exists y \in Y \text { such that }(u, y) \in \operatorname{cl}_{\mu} \Xi_{\Delta}\right\}
$$

Indeed, let $u$ be an arbitrary element of $\bar{\Lambda}$. Then there exists a sequence $\left\{u_{\varepsilon}\right\}_{\varepsilon>0} \in \Lambda$ such that $u_{\varepsilon} \rightarrow u$ in $L^{p}(\Omega)$ as $\varepsilon \rightarrow 0$. By definition of the set $\Lambda$, it follows that we can construct a sequence of pairs $\left\{\left(u_{\varepsilon}, y_{\varepsilon}\right)\right\}_{\varepsilon>0}$ such that $\left(u_{\varepsilon}, y_{\varepsilon}\right) \in \Xi_{\Delta}$ for all $\varepsilon>0$. In view of the definition 
of the set $\Xi_{\Delta}$ and Theorem 3.3, the sequence of states $\left\{y_{\varepsilon}\right\}_{\varepsilon>0}$ is bounded in $H^{1}(\Omega)$. So, we can suppose that there exists an element $y \in H^{1}(\Omega)$ such that, up to a subsequence, $y_{\varepsilon} \rightarrow y$ in $H^{1}(\Omega)$. Hence, $\left(u_{\varepsilon}, y_{\varepsilon}\right) \stackrel{\mu}{\longrightarrow}(u, y)$ in $L^{p}(\Omega) \times H^{1}(\Omega)$ and therefore $(u, y) \in \mathrm{cl}_{\mu} \Xi_{\Delta}$. As a result, we see that $u$ belongs to the set $\left\{v \in L^{p}(\Omega): \exists y \in Y\right.$ such that $\left.(v, y) \in \operatorname{cl}_{\mu} \Xi_{\Delta}\right\}$, that is, we have shown that

$$
\bar{\Lambda} \subseteq\left\{u \in L^{p}(\Omega): \exists y \in Y \text { such that }(u, y) \in \operatorname{cl}_{\mu} \Xi_{\Delta}\right\} .
$$

In order to establish the converse inclusion, we fix an arbitrary pair $\left(u^{*}, y^{*}\right)$ in $\mathrm{cl}_{\mu} \Xi_{\Delta}$. Then $u^{*} \in\left\{u \in L^{p}(\Omega): \exists y \in Y\right.$ s.t. $\left.(u, y) \in \mathrm{cl}_{\mu} \Xi_{\Delta}\right\}$ and there exists a sequence $\left\{\left(u_{\varepsilon}, y_{\varepsilon}\right)\right\}_{\varepsilon>0} \subset L^{p}(\Omega) \times Y$ such that $\left(u_{\varepsilon}, y_{\varepsilon}\right) \in \Xi_{\Delta}$ for each $\varepsilon>0$ and $\left(u_{\varepsilon}, y_{\varepsilon}\right) \stackrel{\mu}{\rightarrow}\left(u^{*}, y^{*}\right)$ in $L^{p}(\Omega) \times H^{1}(\Omega)$. Since the condition $\left(u_{\varepsilon}, y_{\varepsilon}\right) \in \Xi_{\Delta}$ implies that $u_{\varepsilon} \in \Lambda$, it follows that $\left\{u_{\varepsilon}\right\}_{\varepsilon>0} \subset \Lambda$ and $u_{\varepsilon} \rightarrow u^{*}$ in $L^{p}(\Omega)$ as $\varepsilon \rightarrow 0$. Hence, $u^{*} \in \mathrm{cl}_{w-L^{p}(\Omega)} \Lambda$. To conclude, it is enough to apply Mazur's theorem. Thus,

$$
\left\{u \in L^{p}(\Omega): \exists y \in Y \text { such that }(u, y) \in \mathrm{cl}_{\mu} \Xi_{\Delta}\right\} \subseteq \bar{\Lambda}
$$

and combining this fact with (5.2), we arrive at the required equality.

It is clear now that $\operatorname{cl}_{\mu} \Xi_{\Delta} \subseteq \Xi$, where the set $\Xi$ is defined in (4.10). However, we cannot exclude the case when we have $(u, y) \in \Xi$ and $u \in L^{p}(\Omega) \backslash \bar{\Lambda}$. Hence, the validity of the inclusion $\Xi \subseteq \operatorname{cl}_{\mu} \Xi_{\Delta}$ is not a clear issue. So, our next intention is to specify the structure of the set $\mathrm{cl}_{\mu} \Xi_{\Delta}$ and see whether the property $\Xi \subseteq \mathrm{cl}_{\mu} \Xi_{\Delta}$ holds.

Lemma 5.1 Assume that $\Omega$ is a star-shaped domain with respect to some of its interior points $x_{0}$. Assume also that, in addition to the property (2.1), the function $F \in C_{\mathrm{loc}}^{1}(\mathbb{R})$ is such that its derivative $f=F^{\prime}: \Omega \rightarrow(0, \infty)$ is a strictly convex function. Then

$$
\operatorname{cl}_{\mu} \Xi_{\Delta}=\left\{(u, y) \mid \begin{array}{l}
u \in \bar{\Lambda}, \quad y \in Y, \\
\int_{\Omega}[(\nabla y, \nabla \varphi)+y \varphi] d x \\
=\int_{\Omega} f(y) \varphi d x+\int_{\Omega} u \varphi d x, \quad \forall \varphi \in C_{0}^{\infty}\left(\mathbb{R}^{N}\right), \\
\frac{\partial y}{\partial v} \quad \text { on } \partial \Omega, \\
\int_{\Omega}\left[|\nabla y|^{2}+y^{2}\right] d x \\
\leq \frac{2 N}{N-2} \int_{\Omega} F(y) d x-\frac{2}{N-2} \int_{\Omega} u\left(x-x_{0}, \nabla y\right) d x
\end{array}\right\} .
$$

Proof Since the set in the right-hand side of (5.3) can be represented as

$$
\Xi \cap(\bar{\Lambda} \times Y)
$$

and the inclusion $\operatorname{cl}_{\mu} \Xi_{\Delta} \subseteq \Xi \cap(\bar{\Lambda} \times Y)$ is obvious, we concentrate at the proof of the reverse inclusion

$$
\Xi \cap(\bar{\Lambda} \times Y) \subseteq \mathrm{cl}_{\mu} \Xi_{\Delta}
$$

Let $\left(u^{*}, y^{*}\right)$ be an arbitrary representative of the set $\Xi \cap(\bar{\Lambda} \times Y)$. Then $y^{*}$ is a weak solution to the boundary value problem (1.1)-(1.2) with $g=u^{*}$ in the sense of Definition 2.1. Our 
aim is to show that $\left(u^{*}, y^{*}\right) \in \mathrm{cl}_{\mu} \Xi_{\Delta}$. To this end, it is enough to prove the existence of the sequence $\left\{\left(u_{\varepsilon}, y_{\varepsilon}\right)\right\}_{\varepsilon>0}$ in $L^{p}(\Omega) \times H^{1}(\Omega)$ such that

$$
\left(u_{\varepsilon}, y_{\varepsilon}\right) \stackrel{\mu}{\rightarrow}\left(u^{*}, y^{*}\right) \quad \text { in } L^{p}(\Omega) \times H^{1}(\Omega), \quad \text { and } \quad\left(u_{\varepsilon}, y_{\varepsilon}\right) \in \Xi_{\Delta} \quad \forall \varepsilon>0 .
$$

The most natural way to construct such sequences is to apply the procedure of the direct smoothing. Indeed, let us define the elements $y_{\varepsilon} \in H^{1}(\Omega)$ as follows:

$$
y_{\varepsilon}(x)=\frac{1}{\sigma^{N}(\varepsilon)} \int_{\mathbb{R}^{N}} K\left(\frac{x-z}{\sigma(\varepsilon)}\right) \varphi_{\varepsilon}(z) d z
$$

where $\sigma(\varepsilon)>0$ is a positive value such that $\sigma(\varepsilon) \rightarrow 0$ as $\varepsilon \rightarrow 0, K$ is a positive compactly supported smooth function with properties

$$
K \in C_{0}^{\infty}\left(\mathbb{R}^{N}\right), \quad \int_{\mathbb{R}^{N}} K(x) d x=1, \quad \text { and } \quad K(x)=K(-x),
$$

and $\left\{\varphi_{\varepsilon}\right\}_{\varepsilon>0}$ is a sequence in $C_{0}^{\infty}\left(\mathbb{R}^{N}\right)$ such that $\varphi_{\varepsilon} \rightarrow y^{*}$ in $H^{1}(\Omega)$ as $\varepsilon \rightarrow 0$.

Then the property

$$
y_{\varepsilon} \rightarrow y^{*} \quad \text { in } L^{2}(\Omega) \quad \text { and } \quad \nabla y_{\varepsilon} \rightarrow \nabla y^{*} \quad \text { in } L^{2}(\Omega)^{N}
$$

is the direct consequence of the classical properties of smoothing. Moreover, we can suppose that $\frac{\partial y_{\varepsilon}}{\partial \nu}$ has zero trace on $\partial \Omega$ for each $\varepsilon$ small enough, i.e., in view of (5.8) we have: $y_{\varepsilon} \in H^{1}(\Omega)$ and $\frac{\partial y_{\varepsilon}}{\partial \nu}=0$ on $\partial \Omega$ for each $\varepsilon>0$. It remains to note that the parameters $\sigma(\varepsilon)$ can be defined such that $\lim _{\varepsilon \rightarrow 0} \sqrt{\varepsilon} / \sigma^{2}(\varepsilon)=0$. Hence,

$$
\sqrt{\varepsilon} \Delta y_{\varepsilon}=\frac{\sqrt{\varepsilon}}{\sigma^{2}(\varepsilon)}\left[\frac{1}{\sigma^{N}(\varepsilon)} \int_{\mathbb{R}^{N}} \Delta K\left(\frac{x-z}{\sigma(\varepsilon)}\right) \varphi_{\varepsilon}(z) d z\right] \rightarrow 0 \quad \text { in } L^{2}(\Omega) .
$$

Summarizing these properties, we can infer that for a given element $y^{*} \in Y \subset H^{1}(\Omega)$ with $\frac{\partial y^{*}}{\partial v}=0$ on $\partial \Omega$ there exists a sequence $\left\{y_{\varepsilon}\right\}_{\varepsilon>0}$ such that

(a) $y_{\varepsilon} \in Y$, $\frac{\partial y_{\varepsilon}}{\partial v}=0$ on $\partial \Omega$ for each $\varepsilon>0$, and $y_{\varepsilon} \rightarrow y^{*}$ in $H^{1}(\Omega)$ as $\varepsilon \rightarrow 0$.

The smoothing parameter $\sigma(\varepsilon)$ can be defined such that for a given non-negative constant $C_{0}$ we have

(b) $\Delta y_{\varepsilon} \in L^{2}(\Omega)$ for each $\varepsilon>0$ and $\lim _{\varepsilon \rightarrow 0}\left(\varepsilon\left\|\Delta y_{\varepsilon}\right\|_{L^{2}(\Omega)}^{2}\right)=C_{0}$.

Moreover, by Lemma 2.4, we have $f\left(y^{*}\right) \in\left(H^{1}(\Omega)\right)^{*}$. Hence, we can supplement the above properties (a)-(b) by the following one:

(c) $\sup _{\varepsilon>0}\left\|f\left(y_{\varepsilon}\right)\right\|_{\left(H^{1}(\Omega)\right)^{*}}<+\infty$.

Let us define the corresponding controls $\left\{u_{\varepsilon}\right\}_{\varepsilon>0}$ as follows:

$$
u_{\varepsilon}:=-\Delta y_{\varepsilon}+y_{\varepsilon}-f\left(y_{\varepsilon}\right), \quad \forall \varepsilon>0 \text {. }
$$

Since $y_{\varepsilon}$ are the smooth functions, we obviously have $u_{\varepsilon} \in L^{p}(\Omega)$. Moreover, by Proposition 3.1, each of the pairs $\left(u_{\varepsilon}, y_{\varepsilon}\right) \in L^{p}(\Omega) \times Y$ is related by the integral identity

$$
\int_{\Omega}\left(\nabla y_{\varepsilon}, \nabla \varphi\right) d x+\int_{\Omega} y_{\varepsilon} \varphi d x=\int_{\Omega} f\left(y_{\varepsilon}\right) \varphi d x+\int_{\Omega} u_{\varepsilon} \varphi d x, \quad \forall \varphi \in C_{0}^{\infty}\left(\mathbb{R}^{N}\right)
$$


and the inequality

$$
\begin{aligned}
& \left(\frac{N}{2}-1\right) \int_{\Omega}\left|\nabla y_{\varepsilon}\right|^{2} d x+\frac{N}{2} \int_{\Omega}\left|y_{\varepsilon}\right|^{2} d x \\
& \quad \leq N \int_{\Omega} F(y) d x-\int_{\Omega} u_{\varepsilon}\left(x-x_{0}, \nabla y_{\varepsilon}\right) d x .
\end{aligned}
$$

Thus, the sequence $\left\{\left(u_{\varepsilon}, y_{\varepsilon}\right)\right\}_{\varepsilon>0}$ belongs to the set $\Xi_{\Delta} \subset \Xi$. It remains to show that $\left(u_{\varepsilon}, y_{\varepsilon}\right) \stackrel{\mu}{\longrightarrow}\left(u^{*}, y^{*}\right)$ in $L^{p}(\Omega) \times H^{1}(\Omega)$. With that in mind, we make use of the following two functional sequences $\left\{\chi_{\varepsilon, 1}\right\}_{\varepsilon>0} \subset L^{\infty}(\Omega)$ and $\left\{\chi_{\varepsilon, 2}\right\}_{\varepsilon>0} \subset L^{\infty}(\Omega)$, where

$$
\chi_{\varepsilon, 1}(x)=\left\{\begin{array}{ll}
1, & \text { if } u_{\varepsilon}(x) y_{\varepsilon}(x) \geq 0, \\
0, & \text { otherwise }
\end{array} \quad \text { and } \quad \chi_{\varepsilon, 2}(x)=1-\chi_{\varepsilon, 1}(x) \quad \forall \varepsilon>0\right.
$$

Since the functions $u_{\varepsilon}$ and $y_{\varepsilon}$ are smooth enough, it follows that each component $Q$ of the sets $\Omega_{\varepsilon}=\left\{x \in \Omega \mid \chi_{\varepsilon, 1}>0\right\}$ and $\Omega \backslash \Omega_{\varepsilon}$ is 2-connected in the sense of Zhikov (i.e. the implication

$$
v \in W^{1,2}(Q) \text { and } \quad \nabla v=0 \quad \text { a.e. in } Q \quad \Longrightarrow \quad v=\text { const } \quad \text { a.e. in } Q
$$

holds true [19]). As a result, we have $\chi_{\varepsilon, i} \in H^{1}(\Omega)$ (see [19], Lemma 1.4). Hence, we can consider $\chi_{\varepsilon, i} y_{\varepsilon}$ as the test function in the integral identity (5.9). Taking into account that

$$
\nabla\left(\chi_{\varepsilon, i} y_{\varepsilon}\right)=\chi_{\varepsilon, i} \nabla y_{\varepsilon}, \quad \text { a.e. in } \Omega \quad \forall \varepsilon>0, i=1,2
$$

and

$$
\int_{\Omega} y_{\varepsilon} f\left(y_{\varepsilon}\right) d x \leq\left\|f\left(y_{\varepsilon}\right)\right\|_{\left(H^{1}(\Omega)\right)^{*}}\left\|y_{\varepsilon}\right\|_{H^{1}(\Omega)},
$$

we get

$$
\begin{aligned}
\left|\int_{\Omega} u_{\varepsilon} y_{\varepsilon} d x\right| & \leq \int_{\Omega}\left|u_{\varepsilon} y_{\varepsilon}\right| d x=\int_{\Omega}\left(u_{\varepsilon} y_{\varepsilon} \chi_{\varepsilon, 1}-u_{\varepsilon} y_{\varepsilon} \chi_{\varepsilon, 2}\right) d x \\
& \leq 2\left\|y_{\varepsilon}\right\|_{H^{1}(\Omega)}^{2}+2\left\|f\left(y_{\varepsilon}\right)\right\|_{\left(H^{1}(\Omega)\right)^{*}}\left\|y_{\varepsilon}\right\|_{H^{1}(\Omega)} \\
& \leq 2\left(\left\|y_{\varepsilon}\right\|_{H^{1}(\Omega)}+\left\|f\left(y_{\varepsilon}\right)\right\|_{\left(H^{1}(\Omega)\right)^{*}}\right)\left\|y_{\varepsilon}\right\|_{H^{1}(\Omega)}
\end{aligned}
$$

Hence,

$$
\sup _{\varepsilon>0}\left\|u_{\varepsilon}\right\|_{\left(H^{1}(\Omega)\right)^{*}} \leq 2\left(\sup _{\varepsilon>0}\left\|y_{\varepsilon}\right\|_{H^{1}(\Omega)}+\sup _{\varepsilon>0}\left\|f\left(y_{\varepsilon}\right)\right\|_{\left(H^{1}(\Omega)\right)^{*}}\right)<+\infty .
$$

As a result, we deduce that the sequence $\left\{u_{\varepsilon}\right\}_{\varepsilon>0}$ is relatively compact with respect to the weak topology of $\left(H^{1}(\Omega)\right)^{*}$.

Let $u^{0} \in\left(H^{1}(\Omega)\right)^{*}$ be any of its cluster point and let $\left\{u_{\varepsilon_{k}}\right\}_{k \in \mathbb{N}}$ be a subsequence of $\left\{u_{\varepsilon}\right\}_{\varepsilon>0}$ such that

$$
\int_{\Omega} u_{\varepsilon_{k}} \varphi d x \rightarrow\left\langle u^{0}, \varphi\right\rangle_{\left(H^{1}(\Omega)\right)^{*} ; H^{1}(\Omega)} \quad \forall \varphi \in H^{1}(\Omega) \text { as } k \rightarrow \infty .
$$


Our next intension is to show that $u^{0} \in L^{2}(\Omega)$. As follows from (5.10), the sequence $\left\{u_{\varepsilon} y_{\varepsilon}\right\}_{\varepsilon>0}$ is bounded in $L^{1}(\Omega)$. Moreover, the properties (5.8) and (3.13) imply that

$$
\begin{aligned}
& \left|\nabla y_{\varepsilon}\right|^{2}+y_{\varepsilon}^{2} \rightarrow\left|\nabla y^{*}\right|^{2}+\left(y^{*}\right)^{2} \quad \text { in } L^{1}(\Omega), \\
& y_{\varepsilon} f\left(y_{\varepsilon}\right) \rightarrow y^{*} f\left(y^{*}\right) \quad \text { in } L^{1}(\Omega) .
\end{aligned}
$$

Due to this and the the energy equality

$$
\int_{\Omega} u_{\varepsilon} y_{\varepsilon} d x=\int_{\Omega}\left[\left|\nabla y_{\varepsilon}\right|^{2}+y_{\varepsilon}^{2}\right] d x-\int_{\Omega} y_{\varepsilon} f\left(y_{\varepsilon}\right) d x, \quad \forall \varepsilon>0
$$

we can conclude that the sequence $\left\{u_{\varepsilon} y_{\varepsilon}\right\}_{\varepsilon>0}$ is equi-integrable on $\Omega$. Hence, by DunfordPettis Theorem, $\left\{u_{\varepsilon} y_{\varepsilon}\right\}_{\varepsilon>0}$ is a weakly compact sequence in $L^{1}(\Omega)$. So, without loss of generality, we can suppose that there exists a function $v: \Omega \rightarrow \mathbb{R}$ such that

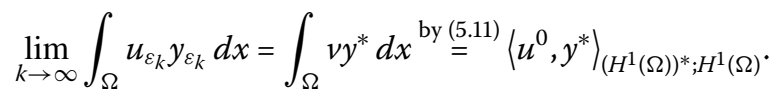

It remains to note that by definition $\left(H^{1}(\Omega)\right)^{*}=H^{-1}(\Omega) \oplus H^{-\frac{1}{2}}(\partial \Omega)$ and $u \in H^{-1}(\Omega)$ if and only if there exists a collection of functions $g_{0}, g_{1}, \ldots, g_{N}$ in $L^{2}(\Omega)$ such that

$$
\langle u, \varphi\rangle_{H^{-1}(\Omega), H_{0}^{1}(\Omega)}=\int_{\Omega} g_{0} \varphi d x-\sum_{i=1}^{N} \int_{\Omega} g_{i} \frac{\partial \varphi}{\partial x_{i}} d x .
$$

Taking into account these facts and comparing them with the representation (5.11), we conclude that the distribution $u^{0} \in\left(H^{1}(\Omega)\right)^{*}$ is regular, $u^{0}=v \in L^{2}(\Omega)$, and

$$
\left\langle u^{0}, y^{*}\right\rangle_{\left(H^{1}(\Omega)\right)^{*} ; H^{1}(\Omega)}=\int_{\Omega} u^{0} y^{*} d x
$$

Thus, in view of equality (5.13) and the fact that $\left\{u_{\varepsilon}\right\}_{\varepsilon>0} \subset L^{2}(\Omega)$, we can suppose that $\left\{u_{\varepsilon}\right\}_{\varepsilon>0}$ is $L^{2}$-bounded sequence and $u_{\varepsilon_{k}} \rightarrow u^{0}$ in $L^{2}(\Omega)$ as $k \rightarrow \infty$.

Since $\left\{\left(u_{\varepsilon_{k}}, y_{\varepsilon_{k}}\right)\right\}_{k \in \mathbb{N}} \subset \Xi_{\Delta}$, it follows that each of the pairs $\left(u_{\varepsilon_{k}}, y_{\varepsilon_{k}}\right)$ is related by the integral identity

$$
\int_{\Omega}\left(\nabla y_{\varepsilon_{k}}, \nabla \varphi\right) d x+\int_{\Omega} y_{\varepsilon_{k}} \varphi d x=\int_{\Omega} f\left(y_{\varepsilon_{k}}\right) \varphi d x+\int_{\Omega} u_{\varepsilon_{k}} \varphi d x, \quad \forall \varphi \in C_{0}^{\infty}\left(\mathbb{R}^{N}\right) .
$$

Taking into account that $y_{\varepsilon_{k}} \rightarrow y^{*}$ in $H^{1}(\Omega)$, we can pass to the limit in (5.14) as $k \rightarrow \infty$ (see the proof of Proposition 3.5 for the details). We get

$$
\int_{\Omega}\left(\nabla y^{*}, \nabla \varphi\right) d x+\int_{\Omega} y^{*} \varphi d x=\int_{\Omega} f\left(y^{*}\right) \varphi d x+\int_{\Omega} u^{0} \varphi d x, \quad \forall \varphi \in C_{0}^{\infty}\left(\mathbb{R}^{N}\right) .
$$

On the other hand, $\left(u^{*}, y^{*}\right) \in \Xi \cap(\bar{\Lambda} \times Y)$. Hence, this pair is related by the similar relation

$$
\int_{\Omega}\left(\nabla y^{*}, \nabla \varphi\right) d x+\int_{\Omega} y^{*} \varphi d x=\int_{\Omega} f\left(y^{*}\right) \varphi d x+\int_{\Omega} u^{*} \varphi d x, \quad \forall \varphi \in C_{0}^{\infty}\left(\mathbb{R}^{N}\right) .
$$


Combining (5.15) with (5.16), we obtain

$$
\int_{\Omega}\left(u^{0}-u^{*}\right) \varphi d x=0, \quad \forall \varphi \in C_{0}^{\infty}\left(\mathbb{R}^{N}\right)
$$

Since $C_{0}^{\infty}\left(\mathbb{R}^{N}\right)$ is dense in $L^{2}(\Omega)$, it follows that $u^{0}=u^{*}$ almost everywhere in $\Omega$. Thus, in view of the fact that $u^{*} \in L^{p}(\Omega)$, we have the same property for $L^{2}$-cluster point $u^{0}$, i.e. $u^{0} \in L^{p}(\Omega)$. It remains to note that this inference is valid for any cluster point $u^{0}$ of the sequence $\left\{u_{\varepsilon}\right\}_{\varepsilon>0}$. Hence, $u^{*}$ is a weak limit in $L^{p}(\Omega)$ for the entire sequence $\left\{u_{\varepsilon}\right\}_{\varepsilon>0}$.

Thus, we have constructed a sequence $\left\{\left(u_{\varepsilon}, y_{\varepsilon}\right)\right\}_{\varepsilon>0}$ in $L^{p}(\Omega) \times H^{1}(\Omega)$ satisfying the properties (5.5). It suffices to conclude that $\left(u^{*}, y^{*}\right) \in \mathrm{cl}_{\mu} \Xi_{\Delta}$. Hence, the inclusion (5.4) is valid. The proof is complete.

As a consequence of this lemma, we can give the following observation.

Proposition 5.2 Under assumptions of Lemma 5.1, we have: for any pair $\left(u^{*}, y^{*}\right) \in \mathrm{cl}_{\mu} \Xi_{\Delta}$ and for any non-negative real number $C_{0}$ there exists a sequence $\left\{\left(\widehat{u}_{\varepsilon}, \widehat{y}_{\varepsilon}\right)\right\}_{\varepsilon>0}$ in $L^{p}(\Omega) \times$ $H^{1}(\Omega)$ such that

$$
\begin{aligned}
& \left(\widehat{u}_{\varepsilon}, \widehat{y}_{\varepsilon}\right) \stackrel{\mu}{\rightarrow}\left(u^{*}, \widehat{y}\right) \quad \text { in } L^{p}(\Omega) \times H^{1}(\Omega), \quad\left(\widehat{u}_{\varepsilon}, \widehat{y}_{\varepsilon}\right) \in \Xi_{\Delta}, \quad \forall \varepsilon>0, \\
& \widehat{u}_{\varepsilon} \rightarrow u^{*} \quad \text { strongly in } L^{p}(\Omega), \\
& \Delta \widehat{y}_{\varepsilon} \in L^{2}(\Omega) \quad \forall \varepsilon>0, \quad \text { and } \quad \lim _{\varepsilon \rightarrow 0}\left(\frac{\varepsilon}{2}\left\|\Delta \widehat{y}_{\varepsilon}\right\|_{L^{2}(\Omega)}^{2}\right)=C_{0},
\end{aligned}
$$

where $\widehat{y}=y^{*}$ provided the Neumann boundary value problem (1.1)-(1.2) has a unique solution for $g=u^{*}$.

Proof Let $\left(u^{*}, y^{*}\right) \in \mathrm{cl}_{\mu} \Xi_{\Delta}$ be an arbitrary pair. Closely following the proof of Lemma 5.1, we can construct a sequence $\left\{\left(u_{\varepsilon_{k}}, y_{\varepsilon_{k}}\right)\right\}_{k \in \mathbb{N}}$ in $L^{p}(\Omega) \times H^{1}(\Omega)$ with properties (5.5) and (a)-(c). Here, $\left\{\varepsilon_{k}\right\}_{k \in \mathbb{N}}$ stands for a strictly decreasing sequence of positive real numbers converging to zero. Then, by Mazur's lemma, there exists a sequence of controls $\left\{\widehat{u}_{\varepsilon_{k}}\right\}_{k \in N}$ such that

$$
\widehat{u}_{\varepsilon_{k}} \rightarrow u^{*} \quad \text { strongly in } L^{p}(\Omega) \quad \text { and } \quad \widehat{u}_{\varepsilon_{k}}=\operatorname{co}\left\{u_{\varepsilon_{1}}, \ldots, u_{\varepsilon_{k}}\right\}, \quad \forall k \in \mathbb{N}
$$

where $\operatorname{co} A$ denotes the convex hull of the set $A$. In view of Lemma 4.2, $\Lambda$ is a convex set. Since $\left\{u_{\varepsilon_{k}}\right\}_{k \in \mathbb{N}} \subset \Lambda$, it follows from (5.20) that $\left\{\widehat{u}_{\varepsilon_{k}}\right\}_{k \in \mathbb{N}} \subset \Lambda$ as well. Let us show that the corresponding weak solutions $\widehat{y}_{k}$ to the boundary value problem

$$
-\Delta y=f(y)+\widehat{u}_{\varepsilon_{k}} \quad \text { in } \Omega, \quad \frac{\partial y}{\partial v}=0 \quad \text { on } \partial \Omega
$$

satisfy properties (5.17) and (5.19).

By definition of the convex hull $\operatorname{co}\left\{u_{\varepsilon_{1}}, u_{\varepsilon_{2}}, \ldots, u_{\varepsilon_{k}}\right\}$ we have: for each $k \in \mathbb{N}$ there exists a collection of non-negative real numbers $\left\{\alpha_{1, k}, \ldots, \alpha_{k, k}\right\}$ such that

$$
\alpha_{1, k}+\alpha_{2, k}+\cdots+\alpha_{k, k}=1 \quad \text { and } \quad \widehat{u}_{\varepsilon}=\alpha_{1, k} u_{\varepsilon_{1}}+\alpha_{2, k} u_{\varepsilon_{2}}+\cdots+\alpha_{k, k} u_{\varepsilon_{k}} .
$$


With each control $\widehat{u}_{\varepsilon_{k}}$ we associate two elements $z_{k} \in H_{0}^{1}(\Omega)$ and $w_{k} \in H_{0}^{1}(\Omega)$ by the following rule:

$$
\begin{aligned}
& -\Delta z_{k}+z_{k}=\widehat{u}_{\varepsilon_{k}}=\sum_{i=1}^{k} \alpha_{i, k} u_{\varepsilon_{i}} \quad \text { in } \Omega \quad \text { and } \quad \frac{\partial z_{k}}{\partial v}=0 \quad \text { on } \partial \Omega, \\
& w_{k}=\alpha_{1, k} y_{\varepsilon_{1}}+\alpha_{2, k} y_{\varepsilon_{2}}+\cdots+\alpha_{k, k} y_{\varepsilon_{k}} .
\end{aligned}
$$

Hence,

$$
-\Delta y_{\varepsilon_{i}}=f\left(y_{\varepsilon_{i}}\right)+u_{\varepsilon_{i}} \quad \text { in } \Omega \quad \text { and } \quad \frac{\partial y_{\varepsilon_{i}}}{\partial v}=0 \quad \text { on } \partial \Omega, i=1, \ldots, k,
$$

imply

$$
-\Delta w_{k}+w_{k}=\sum_{i=1}^{k} \alpha_{i, k} f\left(y_{i, k}\right)+\widehat{u}_{\varepsilon_{k}} \quad \text { in } \Omega \quad \text { and } \quad \frac{\partial w_{k}}{\partial v}=0 \quad \text { on } \partial \Omega .
$$

Taking into account the facts that $f(y)>0$ almost everywhere in $\Omega$ (see (2.1)) and $f$ satisfies Jensen's inequality

$$
f\left(w_{k}\right) \leq \sum_{i=1}^{k} \alpha_{i, k} f\left(y_{i, k}\right)
$$

we obtain

$$
-\Delta z_{k}+z_{k} \leq f\left(z_{k}\right)+\widehat{u}_{\varepsilon_{k}} \quad \text { and } \quad \Delta w_{k}+z_{k} \stackrel{\text { by (5.23) and (5.24) }}{\geq} f\left(w_{k}\right)+\widehat{u}_{\varepsilon_{k}}
$$

i.e. $z_{k}$ is a subsolution to the boundary value problem

$$
-\Delta \psi+\psi=f(\psi)+\widehat{u}_{\varepsilon_{k}} \quad \text { in } \Omega, \quad \frac{\partial \psi}{\partial v}=0 \quad \text { on } \partial \Omega,
$$

and $w_{k}$ is its supersolution. Moreover, since

$$
-\Delta w_{k}+w_{k} \geq f\left(w_{k}\right)+\widehat{u}_{\varepsilon_{k}} \stackrel{\text { by }(2.1)}{\geq} \widehat{u}_{\varepsilon_{k}}=-\Delta z_{k}+z_{k} \quad \text { in } \Omega,
$$

it follows that $-\Delta\left(w_{k}-z_{k}\right)+\left(w_{k}-z_{k}\right) \geq 0$ and $\frac{\partial w_{k}}{\partial v}=0=\frac{\partial z_{k}}{\partial v}$ on $\partial \Omega$. Hence, by the strong maximum principle [17], we conclude that $w_{k} \geq z_{k}$ in $\Omega$. Thus, following the classical techniques [18], we can deduce that for given control $\widehat{u}_{\varepsilon_{k}}$ there exists a solution $\widehat{y}_{\varepsilon_{k}}$ to the above Neumann boundary value problem and it is such that

$$
z_{k}(x) \leq \widehat{y}_{\varepsilon_{k}}(x) \leq w_{k}(x) \quad \text { almost everywhere in } \Omega \text {. }
$$

Moreover, as follows from (5.27) and the fact that $z_{k}, w_{k} \in H^{1}(\Omega)$, we have $\widehat{y}_{\varepsilon_{k}} \in H^{1}(\Omega)$ and

$$
\begin{aligned}
& \int_{\Omega} f\left(\widehat{y}_{\varepsilon_{k}}\right) d x \stackrel{\text { by (5.27) and (2.1) }}{\leq} \int_{\Omega} f\left(\omega_{k}\right) d x \\
& \stackrel{\text { by (5.24) }}{\leq} \sum_{i=1}^{k} \alpha_{i, k}\left\|f\left(y_{i, k}\right)\right\|_{L^{1}(\Omega)}<+\infty .
\end{aligned}
$$


Hence, $\widehat{y}_{\varepsilon_{k}} \in Y$ and, therefore, $\widehat{y}_{\varepsilon_{k}}$ is a weak solution to BVP (5.26).

Let us show that $\left\{\widehat{y}_{\varepsilon_{k}}\right\}_{k \in \mathbb{N}}$ is a weakly compact sequence in $H^{1}(\Omega)$ with the extra property

$$
\Delta \widehat{y}_{\varepsilon_{k}} \in L^{2}(\Omega) \quad \forall \varepsilon>0, \quad \text { and } \quad \lim _{\varepsilon_{k} \rightarrow 0}\left(\frac{\varepsilon_{k}}{2}\left\|\Delta \widehat{y}_{\varepsilon_{k}}\right\|_{L^{2}(\Omega)}^{2}\right)=C_{0} .
$$

Indeed, as follows from (5.21), we have

$$
\begin{aligned}
\sup _{k \in \mathbb{N}}\left\|z_{k}\right\|_{H^{1}(\Omega)} & =\sup _{k \in \mathbb{N}}\left\|(-\Delta+I)^{-1} \widehat{u}_{\varepsilon_{k}}\right\|_{H^{1}(\Omega)} \leq \sup _{k \in \mathbb{N}}\left(\sum_{i=1}^{k} \alpha_{i, k}\left\|u_{\varepsilon_{i}}\right\|_{L^{2}(\Omega)}\right) \\
& \leq|\Omega|^{\frac{p-2}{2 p}} \sup _{k \in \mathbb{N}}\left(\sum_{i=1}^{k} \alpha_{i, k}\left\|u_{\varepsilon_{i}}\right\|_{L^{p}(\Omega)}\right) \\
& \leq|\Omega|^{\frac{p-2}{2 p}} \sup _{k \in \mathbb{N}}\left\|u_{\varepsilon_{k}}\right\|_{L^{p}(\Omega)}<+\infty
\end{aligned}
$$

and

$$
\begin{aligned}
\sup _{k \in \mathbb{N}}\left\|\Delta z_{k}\right\|_{L^{2}(\Omega)} & \leq \sup _{k \in \mathbb{N}}\left\|\widehat{u}_{\varepsilon_{k}}\right\|_{L^{2}(\Omega)}+\sup _{k \in \mathbb{N}}\left\|z_{k}\right\|_{H^{1}(\Omega)} \\
& \leq \sup _{k \in \mathbb{N}}\left[\left(\sum_{i=1}^{k} \alpha_{i, k}\right)\left\|u_{\varepsilon_{k}}\right\|_{L^{2}(\Omega)}\right]+|\Omega|^{\frac{p-2}{2 p}} \sup _{k \in \mathbb{N}}\left\|u_{\varepsilon_{k}}\right\|_{L^{p}(\Omega)} \\
& \leq 2|\Omega|^{\frac{p-2}{2 p}} \sup _{k \in \mathbb{N}}\left\|u_{\varepsilon_{k}}\right\|_{L^{p}(\Omega)}<+\infty
\end{aligned}
$$

As to the supersolutions $w_{k}$, we get

$$
\begin{aligned}
\sup _{k \in \mathbb{N}}\left\|w_{k}\right\|_{H^{1}(\Omega)}= & \sup _{k \in \mathbb{N}}\left\|\sum_{i=1}^{k} \alpha_{i, k} y_{\varepsilon_{i}}\right\| \|_{H^{1}(\Omega)} \\
& \left(\text { by }(3.8) \text { and the fact that }\left(u_{\varepsilon_{k}}, y_{\varepsilon_{k}}\right) \in \Xi_{\Delta}\right) \\
\leq & \sup _{k \in \mathbb{N}}\left[\left(\sum_{i=1}^{k} \alpha_{i, k}\right)\left(C_{4}\left\|u_{\varepsilon_{k}}\right\|_{L^{p}(\Omega)}+C_{5}\right)\right] \\
= & C_{4} \sup _{k \in \mathbb{N}}\left\|u_{\varepsilon_{k}}\right\|_{L^{p}(\Omega)}+C_{5}<+\infty
\end{aligned}
$$

and

$$
\begin{aligned}
\lim _{\varepsilon_{k} \rightarrow 0}\left(\sqrt{\varepsilon_{k}}\left\|\Delta w_{k}\right\|_{L^{2}(\Omega)}\right) & \leq \lim _{\varepsilon_{k} \rightarrow 0}\left(\sqrt{\varepsilon_{k}}\left[\sum_{i=1}^{k} \alpha_{i, k}\left\|\Delta y_{\varepsilon_{i}}\right\|_{L^{2}(\Omega)}\right]\right) \\
& \leq \lim _{\varepsilon_{k} \rightarrow 0}\left[\sqrt{\varepsilon_{k}}\left\|\Delta y_{\varepsilon_{k}}\right\|_{L^{2}(\Omega)}\right] \stackrel{\text { by property (b) }}{=} 0 .
\end{aligned}
$$

As a result, it follows from inequality (5.27) and estimates (5.29), and (5.31) that the sequence $\left\{\widehat{y}_{\varepsilon_{k}}\right\}_{k \in \mathbb{N}}$ is bounded in $H^{1}(\Omega)$ and, hence, there exists an element $\widehat{y} \in H^{1}(\Omega)$ such that, up to a subsequence,

$$
\widehat{y}_{\varepsilon_{k}} \rightarrow \widehat{y} \quad \text { in } H^{1}(\Omega)
$$


Then Proposition 3.5 implies that $\widehat{y}$ is a weak solution to BVP (1.1)-(1.2) for $g=u^{*}$ and the pair $\left(u^{*}, \widehat{y}\right)$ satisfies the inequality (3.1). It is clear now that $\hat{y}=y^{*}$ provided the Neumann boundary value problem (1.1)-(1.2) has a unique solution for $g=u^{*}$. Thus, the sequence $\left\{\left(\widehat{u}_{\varepsilon}, \widehat{y}_{\varepsilon}\right)\right\}_{\varepsilon>0}$ possesses the desired properties (5.17)-(5.18). As to the property (5.19), its validity immediately follows from (5.30) and (5.32). The proof is complete.

Our main intention in this paper is to discuss the variational properties of the solutions to the sequence of fictitious optimal control problems (4.1) as $\varepsilon \rightarrow 0$. As usual, we assume that $\Omega$ is star-shaped with respect to some of its interior point $x_{0}$ and in addition to the property (2.1), the function $f: \Omega \rightarrow(0, \infty)$ is strictly convex. Let $\left\{\left(u_{\varepsilon}^{0}, y_{\varepsilon}^{0}\right)\right\}_{\varepsilon>0} \subset \Xi_{\Delta}$ be a sequence of optimal pairs to the corresponding fictitious problem (4.1). As follows from Theorem 4.4, each of the OCPs (4.1) has a unique solution. We begin with the following result.

Proposition 5.3 There exists a pair $\left(u^{0}, y^{0}\right) \in \mathrm{cl}_{\mu} \Xi_{\Delta}$ such that, within a subsequence,

$$
\begin{aligned}
& \left(u_{\varepsilon}^{0}, y_{\varepsilon}^{0}\right) \stackrel{\mu}{\rightarrow}\left(u^{0}, y^{0}\right) \quad \text { in } L^{p}(\Omega) \times H^{1}(\Omega), \\
& \lim _{\varepsilon \rightarrow 0}\left(\frac{\varepsilon}{2}\left\|\Delta y_{\varepsilon}^{0}\right\|_{L^{2}(\Omega)}^{2}\right)=C_{\Delta}
\end{aligned}
$$

for some $C_{\Delta} \geq 0$.

Proof Since the pair $(\widetilde{u}, \widetilde{y}):=(-f(0), 0)$ is feasible for each of OCPs $(4.1)$, i.e. $(\widetilde{u}, \widetilde{y}) \in \Xi_{\Delta}$, it follows that

$$
J_{\varepsilon}\left(u_{\varepsilon}^{0}, y_{\varepsilon}^{0}\right)=\inf _{(u, y) \in \Xi_{\Delta}} J_{\varepsilon}(u, y) \leq J_{\varepsilon}(\tilde{u}, \tilde{y})=\underbrace{\frac{1}{p}\|g+f(0)\|_{L^{p}(\Omega)}}_{C^{*}}<+\infty, \quad \forall \varepsilon>0 .
$$

Hence,

$$
\begin{aligned}
& \sup _{\varepsilon>0}\left[\frac{\varepsilon}{2}\left\|\Delta y_{\varepsilon}^{0}\right\|_{L^{2}(\Omega)}^{2}\right] \leq C^{*} \quad \text { and } \\
& \sup _{\varepsilon>0}\left\|u_{\varepsilon}^{0}\right\|_{L^{p}(\Omega)}^{p} \leq 2^{p-1}\|g\|_{L^{p}(\Omega)}^{p}+2^{p-1} \sup _{\varepsilon>0}\left\|g-u_{\varepsilon}^{0}\right\|_{L^{p}(\Omega)}^{p} \\
& \leq 2^{p-1}\|g\|_{L^{p}(\Omega)}^{p}+2^{p-1} p \sup _{\varepsilon>0} J_{\varepsilon}\left(u_{\varepsilon}^{0}, y_{\varepsilon}^{0}\right) \\
& \leq 2^{p-1}\|g\|_{L^{p}(\Omega)}^{p}+2^{p-1} p C^{*}, \\
& \sup _{\varepsilon>0}\left\|y_{\varepsilon}^{0}\right\|_{H^{1}(\Omega)} \stackrel{\text { by (3.8) }}{\leq} C_{4} \sup _{\varepsilon>0}\left\|u_{\varepsilon}^{0}\right\|_{L^{p}(\Omega)}+C_{5}<+\infty \text {. }
\end{aligned}
$$

So, we can suppose that there exist a subsequence of $\left\{\left(u_{\varepsilon}^{0}, y_{\varepsilon}^{0}\right)\right\}_{\varepsilon>0}$ (still denoted by the same index $\varepsilon$ ), a pair $\left(u^{0}, y^{0}\right) \in L^{p}(\Omega) \times H^{1}(\Omega)$, and a constant $C_{\Delta} \geq 0$ such that

$$
\lim _{\varepsilon \rightarrow 0}\left[\frac{\varepsilon}{2}\left\|\Delta y_{\varepsilon}^{0}\right\|_{L^{2}(\Omega)}^{2}\right]=C_{\Delta} \quad \text { and } \quad\left(u_{\varepsilon}^{0}, y_{\varepsilon}^{0}\right) \stackrel{\mu}{\rightarrow}\left(u^{0}, y^{0}\right) \quad \text { in } L^{p}(\Omega) \times H^{1}(\Omega) .
$$

To conclude the proof, it remains to note that $\left(u^{0}, y^{0}\right) \in \mathrm{cl}_{\mu} \Xi_{\Delta}$ by Proposition 3.5 and Lemma 5.1. 
The main question arising in this case is about the variational properties of the $\mu$-cluster pairs $\left(u^{0}, y^{0}\right) \in L^{p}(\Omega) \times H^{1}(\Omega)$.

Theorem 5.4 Let $\left(u^{0}, y^{0}\right) \in L^{p}(\Omega) \times H^{1}(\Omega)$ be a $\mu$-cluster pair of the sequence of optimal solutions $\left\{\left(u_{\varepsilon}^{0}, y_{\varepsilon}^{0}\right)\right\}_{\varepsilon>0}$ to the fictitious problems (4.1) as $\varepsilon \rightarrow 0$. Then

$$
J_{0}\left(u^{0}, y^{0}\right)=\inf _{(u, y) \in \mathrm{cl}_{\mu} \Xi_{\Delta}} J_{0}(u, y)
$$

where

$$
J_{0}(u, y)=\frac{1}{p}\|g-u\|_{L^{p}(\Omega)}^{p}, \quad \forall(u, y) \in \mathrm{cl}_{\mu} \Xi_{\Delta} .
$$

Proof To begin with, let us show that the constrained minimization problem

$$
\left\langle\inf _{(u, y) \in \mathrm{c}_{\mu} \Xi_{\Delta}} J_{0}(u, y)\right\rangle
$$

has a non-empty set of solutions. Indeed, in view of definition of the set $\operatorname{cl}_{\mu} \Xi_{\Delta}$ (see Lemma 5.1), there exists a sequence $\left\{\left(u_{k}, y_{k}\right)\right\}_{k \in \mathbb{N}} \subset \operatorname{cl}_{\mu} \Xi_{\Delta}$ such that

$$
\lim _{k \rightarrow \infty} J_{0}\left(u_{k}, y_{k}\right)=\inf _{(u, y) \in \mathrm{cl} \mathrm{l}_{\mu} \Xi_{\Delta}} J_{0}(u, y)
$$

Moreover, because of the density of $C^{\infty}(\bar{\Omega}) \times C_{0}^{\infty}\left(\mathbb{R}^{N}\right)$ in $L^{p}(\Omega) \times H^{1}(\Omega)$, we can suppose that the sequence is rather regular. For our purpose it is enough to have the following inclusion: $\left(u_{k}, y_{k}\right) \in \Xi_{\Delta}$ for all $k \in \mathbb{N}$. Then there exists a constant $C>0$ such that

$$
\begin{aligned}
\sup _{k \in \mathbb{N}}\left\|u_{k}\right\|_{L^{p}(\Omega)}^{p} & \leq 2^{p-1}\|g\|_{L^{p}(\Omega)}^{p}+2^{p-1} \sup _{k \in \mathbb{N}}\left\|g-u_{k}\right\|_{L^{p}(\Omega)}^{p} \\
& \leq 2^{p-1}\|g\|_{L^{p}(\Omega)}^{p}+2^{p-1} p \sup _{k \in \mathbb{N}} J_{0}\left(u_{k}, y_{k}\right) \\
& \stackrel{\text { by (5.37) }}{\leq} 2^{p-1}\|g\|_{L^{p}(\Omega)}^{p}+2^{p-1} p C^{*}, \\
\sup _{k \in \mathbb{N}}\left\|y_{k}\right\|_{H^{1}(\Omega)} & \stackrel{\text { by (3.8) }}{\leq} C_{4} \sup _{k \in \mathbb{N}}\left\|u_{k}\right\|_{L^{p}(\Omega)}+C_{5}<+\infty .
\end{aligned}
$$

So, the minimizing sequence $\left\{\left(u_{k}, y_{k}\right)\right\}_{k \in \mathbb{N}}$ is relatively $\mu$-compact in $L^{p}(\Omega) \times H^{1}(\Omega)$, that is, there exists a pair $\left(u^{*}, y^{*}\right) \in L^{p}(\Omega) \times H^{1}(\Omega)$ such that, up to a subsequence,

$$
\left(u_{k}, y_{k}\right) \stackrel{\mu}{\rightarrow}\left(u^{*}, y^{*}\right) \quad \text { and } \quad\left(u^{*}, y^{*}\right) \stackrel{\text { by Proposition } 3.5 \text { and Lemma } 5.1}{\in} \mathrm{cl}_{\mu} \Xi_{\Delta} \text {. }
$$

To conclude the optimality of $\left(u^{*}, y^{*}\right)$ to the problem $\left\langle\inf _{(u, y) \in \mathrm{cl}_{\mu} \Xi_{\Delta}} J_{0}(u, y)\right\rangle$, it remains to make use of the lower semi-continuity of the cost functional $J_{0}: \mathrm{cl}_{\mu} \Xi_{\Delta} \rightarrow \mathbb{R}$ with respect to the $\mu$-convergence.

We are now in a position to prove the equality (5.36). By contraposition, let us assume that

$$
J_{0}\left(u^{0}, y^{0}\right)>\inf _{(u, y) \in \mathrm{cl}_{\mu} \Xi_{\Delta}} J_{0}(u, y)=J_{0}\left(u^{*}, y^{*}\right) \quad \text { for some }\left(u^{*}, y^{*}\right) \in \mathrm{cl}_{\mu} \Xi_{\Delta} .
$$


Then, due to Proposition 5.2, we can construct a sequence $\left\{\left(\widehat{u}_{\varepsilon}, \widehat{y}_{\varepsilon}\right)\right\}_{\varepsilon>0}$ in $L^{p}(\Omega) \times H^{1}(\Omega)$ with properties

$$
\begin{aligned}
& \left(\widehat{u}_{\varepsilon}, \widehat{y}_{\varepsilon}\right) \stackrel{\mu}{\rightarrow}\left(u^{*}, \widehat{y}\right) \quad \text { in } L^{p}(\Omega) \times H^{1}(\Omega), \quad\left(\widehat{u}_{\varepsilon}, \widehat{y}_{\varepsilon}\right) \in \Xi_{\Delta}, \quad \forall \varepsilon>0, \\
& \widehat{u}_{\varepsilon} \rightarrow u^{*} \quad \text { strongly in } L^{p}(\Omega), \\
& \Delta \widehat{y}_{\varepsilon} \in L^{2}(\Omega) \quad \forall \varepsilon>0, \quad \text { and } \quad \lim _{\varepsilon \rightarrow 0}\left(\frac{\varepsilon}{2}\left\|\Delta \widehat{y}_{\varepsilon}\right\|_{L^{2}(\Omega)}^{2}\right)=C_{\Delta},
\end{aligned}
$$

where the constant $C_{\Delta}$ is defined by (5.34). Moreover, since we have a weak solution to the boundary value problem

$$
-\Delta y=f(y)+u^{*} \quad \text { in } \Omega, \quad \frac{\partial y}{\partial v}=0 \quad \text { on } \partial \Omega
$$

can be non-unique, we admit the case that $y^{*} \neq \widehat{y}$ as elements of $H^{1}(\Omega)$. Then we can write down

$$
J_{\varepsilon}\left(u_{\varepsilon}^{0}, y_{\varepsilon}^{0}\right)=\inf _{(u, y) \in \Xi_{\Delta}} J_{\varepsilon}(u, y) \leq J_{\varepsilon}\left(\widehat{u}_{\varepsilon}, \widehat{y}_{\varepsilon}\right), \quad \forall \varepsilon>0
$$

or in other terms

$$
\frac{\varepsilon}{2} \int_{\Omega}\left|\Delta y_{\varepsilon}^{0}\right|^{2} d x+\frac{1}{p} \int_{\Omega}\left|g-u_{\varepsilon}^{0}\right|^{p} d x \leq \frac{\varepsilon}{2} \int_{\Omega}\left|\Delta \widehat{y}_{\varepsilon}\right|^{2} d x+\frac{1}{p} \int_{\Omega}\left|g-\widehat{u}_{\varepsilon}\right|^{p} d x .
$$

Passing to the limit in both sides of this inequality as $\varepsilon \rightarrow 0$ and taking into account that

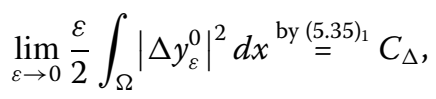

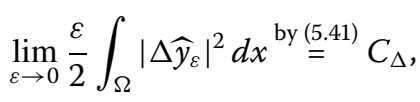

$$
\begin{aligned}
& \liminf _{\varepsilon \rightarrow 0} \int_{\Omega}\left|g-u_{\varepsilon}^{0}\right|^{p} d x \stackrel{\text { by (5.33) }}{\geq} \int_{\Omega}\left|g-u^{0}\right|^{p} d x,
\end{aligned}
$$

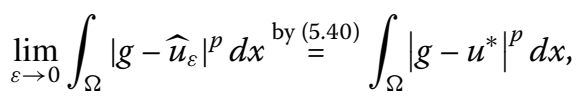

we arrive at the relation

$$
J_{0}\left(u^{0}, y^{0}\right)=\frac{1}{p} \int_{\Omega}\left|g-u^{0}\right|^{p} d x \leq \frac{1}{p} \int_{\Omega}\left|g-u^{*}\right|^{p} d x=J_{0}\left(u^{*}, \widehat{y}\right) \equiv J_{0}\left(u^{*}, y^{*}\right),
$$

which comes into conflict with (5.38). The proof is complete.

\section{On optimality conditions for the fictitious OCPs}

The main goal of this section is to derive some optimality conditions for the fictitious OCP

$$
J_{\varepsilon}(u, y)=\frac{\varepsilon}{2} \int_{\Omega}|\Delta y|^{2} d x+\frac{1}{p} \int_{\Omega}|g-u|^{p} d x \rightarrow \inf
$$


subjected to the constraints

$$
\begin{aligned}
& -\Delta y+y=f(y)+u \quad \text { in } \Omega, \\
& \frac{\partial y}{\partial v}=0 \quad \text { on } \partial \Omega, \\
& u \in L^{p}(\Omega) \quad \text { for some } p>2 .
\end{aligned}
$$

Let $s=\frac{r N}{N+r}$ with $r>N>2$, and let $h \in L^{s}(\Omega)$ be a given distribution. Due to the wellknown regularity result (see [20]), the unique solution $z \in H^{1}(\Omega)$ of the following Neumann boundary value problem:

$$
\begin{aligned}
& -\Delta z+z=h \quad \text { in } \Omega, \\
& \frac{\partial z}{\partial v}=0 \quad \text { on } \partial \Omega,
\end{aligned}
$$

belongs to $L^{\infty}(\Omega)$ and there exists a constant $C_{s}$ (independent of $z$ and $h$ ) such that

$$
\|z\|_{L^{\infty}(\Omega)} \leq C_{s}\|h\|_{L^{s}(\Omega)}
$$

Moreover, since $L^{s}(\Omega)$ is continuously injected into $\left(H^{1}(\Omega)\right)^{*}$, it follows from $(6.7)$ that

$$
\|z\|_{L^{\infty}(\Omega)} \leq C_{s}^{*}\|h\|_{\left(H^{1}(\Omega)\right)^{*}} \quad \text { with some } C_{s}^{*}>0 .
$$

We are now in a position to establish the following result.

Theorem 6.1 Assume that $\Omega$ is a bounded open subset of $\mathbb{R}^{N}(N>2)$ which is star-shaped with respect to some of its interior point $x_{0}$. Let $F: \mathbb{R} \rightarrow(0,+\infty)$ be a mapping of the class $C_{\text {loc }}^{2}(\mathbb{R})$ such that $F$ satisfies estimate $(2.1)$, its derivative $f=F^{\prime}: \mathbb{R} \rightarrow(0, \infty)$ is a strictly convex function, the second derivative $F^{\prime \prime}$ is bounded from below, and $f$ is log-quasi-additive, i.e. there exists a constant $C^{*}>0$ such that

$$
f(v+z) \leq C^{*} f(v) f(z) \quad \forall v, z \in \mathbb{R} .
$$

For a fixed $\varepsilon>0$, let $\left(u_{\varepsilon}^{0}, y_{\varepsilon}^{0}\right) \in \Xi_{\Delta}$ be an optimal pair to the problem (6.1)-(6.4) with an extra property

$$
f\left(y_{\varepsilon}^{0}\right) \in L^{p}(\Omega)
$$

Then there exists a distribution $\varphi_{\varepsilon} \in H^{1}(\Omega)$ such that $\left(u_{\varepsilon}^{0}, y_{\varepsilon}^{0}, \varphi_{\varepsilon}\right) \in L^{p}(\Omega) \times H^{1}(\Omega) \times H^{1}(\Omega)$ satisfies the following optimality system:

$$
\begin{aligned}
& -\Delta y_{\varepsilon}^{0}+y_{\varepsilon}^{0}=f\left(y_{\varepsilon}^{0}\right)+u_{\varepsilon}^{0} \quad \text { in } \Omega, \quad \frac{\partial y_{\varepsilon}^{0}}{\partial v}=0 \quad \text { on } \partial \Omega, \\
& -\Delta \varphi_{\varepsilon}+\left(1-f^{\prime}\left(y_{\varepsilon}^{0}\right)\right) \varphi_{\varepsilon}=\varepsilon \Delta y_{\varepsilon}^{0}\left(1-f^{\prime}\left(y_{\varepsilon}^{0}\right)\right) \quad \text { in } \Omega, \quad \frac{\partial \varphi_{\varepsilon}}{\partial v}=0 \quad \text { on } \partial \Omega, \\
& \varphi_{\varepsilon}=-\left|g-u_{\varepsilon}^{0}\right|^{p-2}\left(g-u_{\varepsilon}^{0}\right)+\varepsilon \Delta y_{\varepsilon}^{0} \quad \text { a.e. in } \Omega .
\end{aligned}
$$


Proof For an arbitrary distribution $z \in H^{1}(\Omega) \cap L^{\infty}(\Omega)$ such that

$$
\frac{\partial z}{\partial \nu}=0 \quad \text { on } \partial \Omega \quad \text { and } \quad \Delta z \in L^{p}(\Omega)
$$

and every $\lambda \in \mathbb{R}(\lambda \neq 0)$, we set

$$
\begin{aligned}
& y_{\varepsilon}^{\lambda}=y_{\varepsilon}^{0}+\lambda z, \\
& u_{\varepsilon}^{\lambda}=u_{\varepsilon}^{0}-\lambda(\Delta z-z)+f\left(y_{\varepsilon}^{0}\right)-f\left(y_{\varepsilon}^{0}+\lambda z\right) .
\end{aligned}
$$

Since

$$
\begin{aligned}
& z \in L^{\infty} \Longrightarrow z \in L^{p}(\Omega) \text { and } \quad(1-f(\lambda z)) \in L^{\infty}(\Omega), \\
& \lambda(\Delta z-z) \stackrel{\text { by (6.14) }}{\in} L^{p}(\Omega), \\
& \left|f\left(y_{\varepsilon}^{0}\right)-f\left(y_{\varepsilon}^{0}+\lambda z\right)\right| \stackrel{\text { by (6.9) }}{\leq}\left(1+C^{*} f(\lambda z)\right) f\left(y_{\varepsilon}^{0}\right) \stackrel{\text { by (6.10) }}{\epsilon} L^{p}(\Omega),
\end{aligned}
$$

it follows from (6.15)-(6.16) that

$$
\begin{aligned}
& u_{\varepsilon}^{\lambda} \in L^{p}(\Omega), \quad \frac{\partial y_{\varepsilon}^{\lambda}}{\partial v}=0 \quad \text { on } \partial \Omega, \quad-\Delta y_{\varepsilon}^{\lambda}+y_{\varepsilon}^{\lambda}=f\left(y_{\varepsilon}^{\lambda}\right)+u_{\varepsilon}^{\lambda} \quad \text { in } \Omega, \\
& \text { and } \quad J_{\varepsilon}\left(u_{\varepsilon}^{\lambda}, y_{\varepsilon}^{\lambda}\right)<+\infty .
\end{aligned}
$$

Hence, $\left(u_{\varepsilon}^{\lambda}, y_{\varepsilon}^{\lambda}\right)$ is a feasible pair to the problem (6.1)-(6.4), i.e. $\left(u_{\varepsilon}^{\lambda}, y_{\varepsilon}^{\lambda}\right) \in \Xi_{\Delta}$ for all values $\lambda \in \mathbb{R}$. As a result, we have the following inequality for the increment of the cost functional:

$$
\Delta J_{\varepsilon}\left(u_{\varepsilon}^{0}, y_{\varepsilon}^{0}\right):=J_{\varepsilon}\left(u_{\varepsilon}^{\lambda}, y_{\varepsilon}^{\lambda}\right)-J_{\varepsilon}\left(u_{\varepsilon}^{0}, y_{\varepsilon}^{0}\right) \geq 0, \quad \forall \lambda \in \mathbb{R}
$$

Using the fact that

$$
\begin{aligned}
\lim _{\lambda \rightarrow 0} & \frac{J_{\varepsilon}\left(u_{\varepsilon}^{\lambda}, y_{\varepsilon}^{\lambda}\right)-J_{\varepsilon}\left(u_{\varepsilon}^{0}, y_{\varepsilon}^{0}\right)}{\lambda} \\
\quad & \frac{\varepsilon}{2} \lim _{\lambda \rightarrow 0} \int_{\Omega} \frac{\left|\Delta y_{\varepsilon}^{\lambda}\right|^{2}-\left|\Delta y_{\varepsilon}^{0}\right|^{2}}{\lambda} d x+\frac{1}{p} \lim _{\lambda \rightarrow 0} \int_{\Omega} \frac{\left|g-u_{\varepsilon}^{\lambda}\right|^{p}-\left|g-u_{\varepsilon}^{0}\right|^{p}}{\lambda} d x \\
\quad & \varepsilon \int_{\Omega} \Delta y_{\varepsilon}^{0} \Delta z d x+\int_{\Omega}\left|g-u_{\varepsilon}^{0}\right|^{p-2}\left(g-u_{\varepsilon}^{0}\right)\left(-\Delta z+z-f^{\prime}\left(y_{\varepsilon}^{0}\right) z\right) d x,
\end{aligned}
$$

we deduce from (6.18) that

$$
\begin{aligned}
& \int_{\Omega}\left(\left|g-u_{\varepsilon}^{0}\right|^{p-2}\left(g-u_{\varepsilon}^{0}\right)-\varepsilon \Delta y_{\varepsilon}^{0}\right)(-\Delta z+z) d x \\
& \quad=-\varepsilon \int_{\Omega} \Delta y_{\varepsilon}^{0} z d x+\int_{\Omega}\left|g-u_{\varepsilon}^{0}\right|^{p-2}\left(g-u_{\varepsilon}^{0}\right) f^{\prime}\left(y_{\varepsilon}^{0}\right) z d x
\end{aligned}
$$

for every $z \in H^{1}(\Omega) \cap L^{\infty}(\Omega)$ with properties (6.14).

Having set

$$
\varphi_{\varepsilon}:=-\left|g-u_{\varepsilon}^{0}\right|^{p-2}\left(g-u_{\varepsilon}^{0}\right)+\varepsilon \Delta y_{\varepsilon}^{0},
$$


we see from (6.5), (6.6), and (6.19) that

$$
\begin{aligned}
\int_{\Omega} \varphi_{\varepsilon} h d x & =\int_{\Omega} \varphi_{\varepsilon}(-\Delta z+z) d x \\
& =\varepsilon \int_{\Omega} \Delta y_{\varepsilon}^{0} z d x-\int_{\Omega}\left|g-u_{\varepsilon}^{0}\right|^{p-2}\left(g-u_{\varepsilon}^{0}\right) f^{\prime}\left(y_{\varepsilon}^{0}\right) z d x .
\end{aligned}
$$

Since we have the convexity inequality

$$
f\left(y_{\varepsilon}^{0}+\lambda z\right)-f\left(y_{\varepsilon}^{0}\right) \geq \lambda f^{\prime}\left(y_{\varepsilon}^{0}\right) z
$$

estimate (6.17), and the boundedness of $f^{\prime}$ from below on $\mathbb{R}$ imply

$$
f^{\prime}\left(y_{\varepsilon}^{0}\right) \in L^{p}(\Omega)
$$

it follows that

$$
\begin{aligned}
\left|\int_{\Omega}\right| g-\left.u_{\varepsilon}^{0}\right|^{p-2}\left(g-u_{\varepsilon}^{0}\right) f^{\prime}\left(y_{\varepsilon}^{0}\right) z d x \mid & \leq \int_{\Omega}\left|g-u_{\varepsilon}^{0}\right|^{p-1}\left|f^{\prime}\left(y_{\varepsilon}^{0}\right)\right| d x\|z\|_{L^{\infty}(\Omega)} \\
& \leq\left\|g-u_{\varepsilon}^{0}\right\|_{L^{p}(\Omega)}^{p-1}\left\|f^{\prime}\left(y_{\varepsilon}^{0}\right)\right\|_{L^{p}(\Omega)}\|z\|_{L^{\infty}(\Omega)} .
\end{aligned}
$$

Moreover, taking into account that

$$
\begin{aligned}
\int_{\Omega} \Delta y_{\varepsilon}^{0} z d x & =\int_{\Omega}\left[y_{\varepsilon}^{0}-f\left(y_{\varepsilon}^{0}\right)-u_{\varepsilon}^{0}\right] z d x \\
& \leq\left[|\Omega|^{\frac{1}{2}}\left\|y_{\varepsilon}^{0}\right\|_{H^{1}(\Omega)}+\left\|f\left(y_{\varepsilon}^{0}\right)\right\|_{L^{1}(\Omega)}+|\Omega|^{\frac{p-1}{p}}\left\|u_{\varepsilon}^{0}\right\|_{L^{p}(\Omega)}\right]\|z\|_{L^{\infty}(\Omega)},
\end{aligned}
$$

we finally get

$$
\int_{\Omega} \varphi_{\varepsilon} h d x \leq B\|z\|_{L^{\infty}(\Omega)} \stackrel{\text { by }(6.8)}{\leq} C_{s}^{*} B\|h\|_{\left(H^{1}(\Omega)\right)^{*}}
$$

where

$$
\begin{aligned}
B= & |\Omega|^{\frac{1}{2}}\left\|y_{\varepsilon}^{0}\right\|_{H^{1}(\Omega)}+\left\|f\left(y_{\varepsilon}^{0}\right)\right\|_{L^{1}(\Omega)}+|\Omega|^{\frac{p-1}{p}}\left\|u_{\varepsilon}^{0}\right\|_{L^{p}(\Omega)} \\
& +\left\|g-u_{\varepsilon}^{0}\right\|_{L^{p}(\Omega)}^{p-1}\left\|f^{\prime}\left(y_{\varepsilon}^{0}\right)\right\|_{L^{p}(\Omega)} .
\end{aligned}
$$

Since the estimate (6.22) is valid for all $h \in C_{0}^{\infty}\left(\mathbb{R}^{N}\right)$ and the set $C_{0}^{\infty}\left(\mathbb{R}^{N}\right)$ is dense in $\left(H^{1}(\Omega)\right)^{*}$, we deduce from the above inequality that $\varphi_{\varepsilon} \in H^{1}(\Omega)$. It remains to notice that equations (6.12) are direct consequences of the integral identity (6.20).

\section{Conclusion}

Because of ill-posedness of the original BVP (1.1)-(1.2), it is reasonably to suppose that the set of solutions to minimization problem (5.36) is not singleton. On the other hand, we have

$$
\inf _{(u, y) \in \mathrm{cl}_{\mu} \Xi_{\Delta}} J_{0}(u, y)=\frac{1}{p} \inf _{(u, y) \in \mathrm{cl}_{\mu} \Xi_{\Delta}}\left[\|g-u\|_{L^{p}(\Omega)}^{p}\right]=\frac{1}{p} \inf _{u \in \bar{\Lambda}}\left[\|g-u\|_{L^{p}(\Omega)}^{p}\right] .
$$


It means that the minimal value of the cost functional $J_{0}\left(u^{0}, y^{0}\right)$ does not depend on the $y$-component of the optimal pair. Hence, Theorem 5.4 implies that even if the sequence of optimal solutions $\left\{\left(u_{\varepsilon}^{0}, y_{\varepsilon}^{0}\right)\right\}_{\varepsilon>0}$ to the fictitious problems (4.1) has more than one $\mu$-cluster pair, their $u$-components must coincide. In other words, if $\left\{\left(u_{\varepsilon}^{0}, y_{\varepsilon}^{0}\right)\right\}_{\varepsilon>0}$ is a sequence of optimal pairs to the fictitious problems (4.1), then $\left\{y_{\varepsilon}^{0}\right\}_{\varepsilon>0}$ is relatively weakly compact in $H^{1}(\Omega)$ and there exists a unique $u^{0} \in \bar{\Lambda}$ such that

$$
u_{\varepsilon}^{0} \rightarrow u^{0} \quad \text { in } L^{p}(\Omega) \text { as } \varepsilon \rightarrow 0,
$$

where $u^{0}$ is a minimizer to the minimization problem $\frac{1}{p} \inf _{u \in \bar{\Lambda}}\left[\|g-u\|_{L^{p}(\Omega)}^{p}\right]$. This circumstance and the ill-posedness of the BVP (1.1)-(1.2) motivates us to introduce the following concept.

Definition 7.1 Let $g$ be a given element of $L^{p}(\Omega)$ with $p>2$. Then we say that a distribution $y^{*}=y^{*}(g) \in H^{1}(\Omega)$ is an approximate solution to the boundary value problem (1.1)-(1.2) if $y^{*} \in Y, \frac{\partial y^{*}}{\partial \nu}=0$ on $\partial \Omega$, and $y^{*}$ satisfies the relations

$$
\begin{aligned}
& \int_{\Omega}\left(\nabla y^{*}, \nabla \varphi\right) d x+\int_{\Omega} y^{*} \varphi d x=\int_{\Omega} f\left(y^{*}\right) \varphi d x+\int_{\Omega} g^{*} \varphi d x, \quad \forall \varphi \in C_{0}^{\infty}\left(\mathbb{R}^{N}\right), \\
& \int_{\Omega}\left|\nabla y^{*}\right|^{2} d x+\int_{\Omega}\left|y^{*}\right|^{2} d x \leq \frac{2 N}{N-2} \int_{\Omega} F\left(y^{*}\right) d x-\frac{2}{N-2} \int_{\Omega} g^{*}\left(x-x_{0}, \nabla y^{*}\right) d x,
\end{aligned}
$$

where

$$
\left\|g-g^{*}\right\|_{L^{p}(\Omega)}^{p}=\inf _{(u, y) \in \mathrm{cl}_{\mu} \Xi_{\Delta}}\|g-u\|_{L^{p}(\Omega)}^{p}
$$

As immediately follows from this definition, the approximate solution $y^{*}$ is not unique, in general. Moreover, $y^{*}$ coincides with a weak solution to (1.1)-(1.2) in the sense of Definition 2.1 provided $g^{*}=g$. However, in this case we have a weak solution with an extra property: this solution satisfies the inequality (3.1) even if we do not know whether $\Delta y^{*} \in L^{2}(\Omega)$. In the context of Definition 7.1, the question arises as to the existence and attainability of the approximate solutions to the boundary value problem (1.1)-(1.2). In view of this, it makes sense to give the following final result which is an obvious consequence of Theorem 5.4, Proposition 5.3, and Theorem 4.4.

Theorem 7.2 Let $\Omega$ be a bounded open subset of $\mathbb{R}^{N}(N>2)$ which is assumed to be starshaped with respect to some of its interior point $x_{0}$. Let $F: \mathbb{R} \rightarrow(0,+\infty)$ be a mapping of the class $C_{\mathrm{loc}}^{1}(\mathbb{R})$ such that $F$ satisfies estimate (2.1) and its derivative $f=F^{\prime}: \mathbb{R}^{N} \rightarrow(0, \infty)$ is a strictly convex function. Let $g \in L^{2}(\Omega)$ be a given distribution. If there exists a positive value $\delta>0$ such that $g \in L^{2+\delta}(\Omega)$, then the set of approximate solutions to the boundary value problem (1.1)-(1.2) is non-empty. Moreover, in this case some of such solutions $y^{*} \in Y$ can be attained as follows: $y^{*}$ is an $H^{1}(\Omega)$-weak cluster point of the sequence $\left\{y_{\varepsilon}^{0}\right\}_{\varepsilon>0}$, where $\left\{\left(u_{\varepsilon}^{0}, y_{\varepsilon}^{0}\right)\right\}_{\varepsilon>0}$ are minimizers to the corresponding fictitious OCPs (4.1). 
Authors' contributions

All authors contributed equally to the writing of this paper. All authors read and approved the final manuscript.

\section{Author details}

'Department of Differential Equations, Dnipropetrovsk National University, Gagarin av., 72, 49010 Dnipro, Ukraine. ${ }^{2}$ Department of Information Engineering, Electrical Engineering and Applied Mathematics, University of Salerno, Via Giovanni Paolo II, 132, Fisciano (SA), Italy.

\section{Acknowledgements}

The authors are very grateful to the referees for their careful reading, comments, and suggestions, which improved the presentation of this paper. This work is partially supported by the FARB 2015 Project entitled 'Modelli Matematici Multidisciplinari Basati su Equazioni Differenziali alle Derivate Parziali'.

Received: 9 August 2016 Accepted: 15 November 2016 Published online: 25 November 2016

\section{References}

1. Gelfand, IM: Some problems in the theory of quasi-linear equations. Transl. Am. Math. Soc. 29, 289-292 (1963)

2. Crandall, MG, Rabinowitz, PH: Some continuation and variational methods for positive solutions of nonlinear elliptic eigenvalue problems. Arch. Ration. Mech. Anal. 58, 207-218 (1975)

3. Mignot, F, Puel, JP: Sur une classe de problèmes non linéaires avec nonlinéarité positive, croissante, convexe. Commun. Partial Differ. Equ., 5 791-836 (1980)

4. Gallouët, T, Mignot, F, Puel, JP: Quelques résultats sur le problème $-\Delta u=\lambda e^{u}$. C. R. Acad. Sci., Ser. 1 Math. 307 289-292 (1988)

5. Fujita, H: On the blowing up of the solutions to the Cauchy problem for $u_{t}=\Delta u+u^{1+\alpha}$. J. Fac. Sci., Univ. Tokyo, Sect. 1A, Math. 13, 109-124 (1996)

6. Pinsky, RG: Existence and nonexistence of global solutions $u_{t}=\Delta u+a(x) u^{p}$ in $\mathbb{R}^{d}$. J. Differ. Equ. 133, 152-177 (1997)

7. Ferreira, R, De Pablo, A, Vazquez, JL: Classification of blow-up with nonlinear diffusion and localized reaction. J. Differ. Equ., 231 195-211 (2006)

8. Dolbeault, J, Stańczy, R: Non-existence and uniqueness results for supercritical semilinear elliptic equations. Ann. Henri Poincaré 10(7), 1311-1333 (2010)

9. Chandrasekhar, S: An Introduction to the Study of Stellar Structures. Dover Publishing Inc., New York (1985)

10. Franck-Kamenetskii, DA: Diffusion and Heat Transfer in Chemical Kinetics, 2nd edn. Plenum Press, New York (1969)

11. Casas, E, Kavian, O, Puel, JP: Optimal control of an ill-posed elliptic semilinear equation with an exponential nonlinearity. ESAIM: Control, Optimization and Calculus of Variations 3, 361-380 (1998)

12. Kinderlehrer, D, Stampacchia, G: An Introduction to Variational Inequalities and Their Applications. Academic Press, New York (1980)

13. Evans, LC: Partial Differential Equations. Graduate Studies in Mathematics, vol. 19. AMS, Providence (2010)

14. Boccardo, L, Murat, F: Almost everywhere convergence of the gradients of solutions to elliptic and parabolic equations. Nonlinear Anal. 19, 581-597 (1992)

15. Fursikov, AV: Optimal Control of Distributed Systems. Theory and Applications. AMS, Providence, RI (2000)

16. Vázquez, JL: A strong maximum principle for some quasilinear elliptic equations. Appl. Math. Optim. 12(1), 191-202 (1984)

17. Friedman, A: Partial Differential Equations of Parabolic Type. Prentice-Hall, New York (1964)

18. Sattinger, DH: Monotone methods in nonlinear elliptic and parabolic boundary value problems. Indiana Univ. Math. J. 21, 979-1000 (1972)

19. Zhikov, W: Connectedness and homogenization. Examples of fractal conductivity. Sbornik: Mathematics 187(8), 1109-1147 (1996)

20. Troianiello, GM: Elliptic Differential Equations and Obstacle Problems. Plenum Press, New York (1987)

\section{Submit your manuscript to a SpringerOpen ${ }^{\circ}$ journal and benefit from:}

- Convenient online submission

Rigorous peer review

- Immediate publication on acceptance

- Open access: articles freely available online

- High visibility within the field

- Retaining the copyright to your article 